\title{
Detecting a Theta or a Prism
}

\author{
Maria Chudnovsky ${ }^{1}$ \\ Columbia University, New York, NY 10027 \\ Rohan Kapadia ${ }^{2}$ \\ McGill University, Montreal, Canada
}

May 16, 2006; revised July 18, 2007

\footnotetext{
${ }^{1}$ This research was conducted during the period the author served as a Clay Mathematics Institute Research Fellow at Princeton University.

${ }^{2}$ This research was conducted at Princeton University
} 


\begin{abstract}
A theta in a graph is an induced subgraph consisting of two nonadjacent vertices joined by three disjoint paths. A prism in a graph is an induced subgraph consisting of two disjoint triangles joined by three disjoint paths. This paper gives a polynomial-time algorithm to test whether a graph has an induced subgraph that is either a prism or a theta.
\end{abstract}




\section{Introduction}

The vertex set of a graph $G$ is denoted by $V(G)$ and the edge set by $E(G)$. All graphs in this paper are simple. A cycle is a connected subgraph in which all vertices have degree two. A path $P$ in $G$ is an induced connected subgraph of $G$ such that either $P$ is a one-vertex graph, or two vertices of $P$ have degree one and all others have degree two. (Please note that we are using a non-standard definition.) The ends of a path are the two vertices with degree one in the path. If $X \subseteq V(G)$, the subgraph with vertex set $X$ and edge set all edges of $G$ with both ends in $X$ is denoted $G \mid X$, and called the subgraph induced on $X$.

A prism is a graph consisting of two disjoint triangles $\left\{a_{1}, a_{2}, a_{3}\right\}$ and $\left\{b_{1}, b_{2}, b_{3}\right\}$ together with three paths $P_{1}, P_{2}$ and $P_{3}$ such that the union of every two of $P_{1}, P_{2}$ and $P_{3}$ is an induced cycle. A theta is a graph consisting of two nonadjacent vertices $s$ and $t$, and three paths $P, Q$ and $R$ with ends $s$ and $t$, such that the union of every two of $P, Q$ and $R$ is an induced cycle. The vertices $s, t$ are called the ends of the theta. Finally, a pyramid is a graph consisting of a triangle $\left\{b_{1}, b_{2}, b_{3}\right\}$, a vertex $a \notin\left\{b_{1}, b_{2}, b_{3}\right\}$, and, for $i=1,2,3$ a path $P_{i}$ between $a$ and $b_{i}$, such that $a$ is adjacent to at most one of $b_{1}, b_{2}$ and $b_{3}$ and the union of every two of $P_{1}, P_{2}$ and $P_{3}$ is an induced cycle. An induced subgraph $H$ of $G$ is a prism, theta or pyramid in $G$ if $H$ is a prism, theta or pyramid, respectively. We say that a graph $G$ contains a prism, a theta or a pyramid, if some induced subgraph of $G$ is a prism, a theta or a pyramid, respectively. A hole in a graph is an induced cycle of length at least four. A hole is odd if it has an odd number of vertices, and even otherwise. Given a graph $G$, the complement $\bar{G}$ of $G$ is the graph with the same vertex set as $G$, and such that two vertices are adjacent in $\bar{G}$ if and only if they are non-adjacent in $G$. A graph $G$ is called Berge if neither $G$ nor $\bar{G}$ contains an odd hole.

In [1] Chudnovsky, Cornuéjols, Liu, Seymour, and Vušković present a polynomial time algorithm to test whether a graph contains a pyramid. The main result of [1] is a polynomial time algorithm to test if a graph is Berge. Since every pyramid contains an odd hole, no Berge graph contains a pyramid, and testing for pyramids is the first step in testing for Bergeness. Similarly to pyramids, every theta and every prism contains an even hole, and therefore a graph with no even holes contains no theta or prism. In [4] Maffray and Trotignon prove that the problem of deciding if a graph contains a prism is NP-complete, and in [2] Chudnovsky and Seymour show that testing if a graph contains a theta can be done in polynomial time. However, the complexity of deciding if a graph contains a prism or a theta 
remained open (and this is the analogue of the pyramid problem for graphs with no even holes). Our main result here is a polynomial time algorithm that given a graph $G$, decides if $G$ contains either a prism or a theta. The algorithm is described in Section 5, and its running time is $O\left(|V(G)|^{35}\right)$.

Before continuing with the outline of this algorithm, we need a few more definitions. The interior of a path $P$ is the set of vertices that have degree two in $P$, and is denoted by $P^{*}$. If $u$ and $v$ are two vertices in a path $P$, then $u-P-v$ denotes the subpath of $P$ whose ends are $u$ and $v$, and if $u$ and $v$ are consecutive then we may denote $u-P-v$ by just $u-v$. For a subgraph $X$ of $G$ we say that $v \in V(G) \backslash V(X)$ has a neighbor in $X$ if there exists a vertex in $V(X)$ adjacent to $v$.

For $A \subseteq V(G)$, a vertex is called $A$-complete if it is adjacent to every vertex in $A$. Two subsets $A, B \subseteq V(G)$ are anticomplete to each other if no vertex of $A$ is adjacent to any vertex of $B$. For any two vertices $a$ and $b$, let $N(a)$ be the set of neighbors of $a$ in $G$, and let $N(a, b)$ be the set of all $\{a, b\}$-complete vertices in $G$.

Let $K$ be a prism. Label the triangles of $K\left\{a_{1}, a_{2}, a_{3}\right\}$ and $\left\{b_{1}, b_{2}, b_{3}\right\}$ and for $i=1,2,3$ let $P_{i}$ be the path from $a_{i}$ to $b_{i}$. For $i=1,2,3$ let $m_{i}$ be the vertex in $P_{i}$ such that for the paths $a_{i}-P_{i}-m_{i}$, denoted by $S_{i}$, and $m_{i}-P_{i}-b_{i}$, denoted by $T_{i}$, we have $\left|E\left(T_{i}\right)\right| \leq\left|E\left(S_{i}\right)\right| \leq\left|E\left(T_{i}\right)\right|+1$. A $K$-major vertex, or just a major vertex when there is no danger of confusion, is a vertex not in $V(K)$ whose neighbors in $K$ are not a subset of the vertex set of a 3 -vertex path contained in $K$.

Finally, a subgraph $H$ is smaller than a subgraph $H^{\prime}$ if $|V(H)|<$ $\left|V\left(H^{\prime}\right)\right|$. An induced subgraph $X$ of $G$ that is either a theta or a prism is called smallest if no other theta or prism in $G$ is smaller than $X$.

The idea of the algorithm is as follows. Let $G$ be the graph input to the algorithm and suppose there is a prism $K$ in $G$. A shortest path $Q$ with the same ends as $S_{1}$ is called a shortcut across $K$. If the subgraph induced by $V(K) \cup Q^{*} \backslash S_{1}^{*}$ is also a prism, then $Q$ is good, and if not then $Q$ is bad. Shortest paths with the same ends as one of $T_{1}, S_{2}, T_{2}, S_{3}$ and $T_{3}$ are also called shortcuts across $K$. Shortcuts will be defined in the same way for thetas. A theta or prism is clean if all shortcuts across it are good. The algorithm uses a procedure called cleaning, first introduced by Conforti and Rao in [3]. The idea of cleaning is to identify a cleaner, which is a subset $X \subseteq V(G)$ such that if $G$ contains a prism or a theta, then the graph $G^{\prime}$ induced by $G$ on $V(G) \backslash X$ contains a clean prism or a clean theta.

In $G^{\prime}$, it becomes easy to detect the clean theta or prism. For example, if $G^{\prime}$ contains a clean prism $K$, then for any shortcut $Q$ across the path $S_{1}$ of $K$, the subgraph induced on $V(K) \cup Q^{*} \backslash S_{1}^{*}$ is also a prism, because $Q$ is 
a good shortcut. The same is true for any other shortcut across $K$. So the algorithm checks all 9-tuples of vertices $a_{1}, a_{2}, a_{3}, m_{1}, m_{2}, m_{3}, b_{1}, b_{2}, b_{3}$; for each of the pairs $a_{1} m_{1}, a_{2} m_{2}, a_{3} m_{3}, m_{1} b_{1}, m_{2} b_{2}, m_{3} b_{3}$, it finds a shortest path joining the pair, then tests whether the subgraph induced by the union of these paths is a prism. If there is a clean smallest prism in $G^{\prime}$, then it can be shown that a prism is found when considering one of the 9-tuples. A similar procedure works to find clean smallest thetas.

There are three stages of cleaning required. The first two cleaning algorithms output a list of polynomially many subsets of $V(G)$, one of which is a cleaner if $G$ contains a smallest prism or theta. The first outputs a cleaner when $G$ contains a smallest theta, and is given in Section 2. The second outputs a cleaner when $G$ contains a smallest prism $K$, and is given in Section 3. However, the second algorithm requires that the input graph $G$ contain no $K$-major vertices. For this reason, we need the third cleaning algorithm, which outputs a list of subsets of which one contains all $K$-major vertices and is disjoint from $V(K)$. This algorithm is given in Section 4 . Finally, the algorithms for detecting clean prisms and thetas are described in Section 5.

\section{Theta shortcuts}

Let $K$ be a theta in $G$ with ends $a$ and $b$ and paths $P_{1}, P_{2}$ and $P_{3}$ between them. For $1 \leq i \leq 3$, let $s_{i}$ be the neighbor of $a$ in $P_{i}$ and $t_{i}$ the neighbor of $b$ in $P_{i}$. Also, for $1 \leq i \leq 3$ define $m_{i} \in P_{i}^{*}$ and subpaths $S_{i}$ and $T_{i}$ of $P_{i}$ such that $S_{i}=s_{i}-P_{i}-m_{i}, T_{i}=m_{i}-P_{i}$ - $t_{i}$, and $\left|V\left(T_{i}\right)\right| \leq\left|V\left(S_{i}\right)\right| \leq\left|V\left(T_{i}\right)\right|+1$. We call $P_{1}, P_{2}$ and $P_{3}$ the paths of $K$ and $S_{1}, T_{1}, S_{2}, T_{2}, S_{3}$, and $T_{3}$ the half-paths of $K$. The frame of the theta $K$ is the 11-tuple

$$
\left\{a, b, s_{1}, s_{2}, s_{3}, m_{1}, m_{2}, m_{3}, t_{1}, t_{2}, t_{3}\right\}
$$

and we say that $K$ has a tidy frame if

- $a$ and $b$ have no neighbors in $V(G) \backslash V(K)$, and

- for $i=1,2,3$, if $m_{i}$ is adjacent to $a$ and $b$ then $m_{i}$ has no neighbors in $V(G) \backslash V(K)$.

If $S$ is one of the half-paths of $K$, then a shortcut across $S$ is a shortest path in $G$ with the same ends as $S$. A shortcut across $K$ is a shortcut across any half-path of $K$. A shortcut $S^{\prime}$ across $S$ is good if no interior vertex of $S^{\prime}$ has neighbors in $V(K) \backslash V(S)$, and bad otherwise. 
2.1. Let $K$ be a smallest theta in $G$. Assume $K$ has a tidy frame. Then there is a vertex $c \in P_{2}^{*} \cup P_{3}^{*}$ such that $c$ has a neighbor in the interior of every bad shortcut across $S_{1}$ or $T_{1}$.

Proof. Let $C$ be the cycle induced by $G$ on $V\left(P_{2}\right) \cup V\left(P_{3}\right)$. Let $Q$ be a bad shortcut across $S_{1}$. Suppose there is no edge between $V(Q)$ and $V(C)$ except $s_{1}-a$. Since $Q$ is a bad shortcut, it follows that some vertex of $Q$ has a neighbor in $V\left(T_{1}\right) \backslash\left\{m_{1}\right\}$ and then the graph induced by $G$ on $V(C) \cup V(Q) \cup V\left(T_{1}\right)$ contains a theta smaller than $K$, a contradiction. Since $m_{1}$ has no neighbors in $C$ and $a$ is the only neighbor of $s_{1}$ in $C$, this proves that some vertex in $Q^{*}$ has a neighbor in $C$. Let $q \in Q^{*}$ be the vertex with neighbors in $C$ such that no other vertex in $q-Q-m_{1}$ has neighbors in $C$.

(1) The vertex $q$ has exactly two neighbors in $C$, and they are adjacent to each other.

If $m_{1}=t_{1}$, then any shortcut between $m_{1}$ and $s_{1}$ has empty interior, and therefore is a good shortcut. Consequently, we may assume $m_{1} \neq t_{1}$.

Suppose $q$ has two neighbors in $C$ that are not adjacent to each other. Let $u$ and $v$ be the neighbors of $q$ in $C$ such that a path between $u$ and $v$ in $C$ contains $b$ and no other neighbor of $q$. Then $u$ and $v$ are not adjacent to each other. Let $C_{u}$ be the path of $C$ from $u$ to $b$ such that $v \notin V\left(C_{u}\right)$ and let $C_{v}$ be the path of $C$ from $v$ to $b$ such that $u \notin V\left(C_{v}\right)$. The paths $q-u-C_{u}-b, q-v-C_{v^{-}} b$ and a path between $q$ and $b$ with interior in $V\left(q-Q-m_{1}-T_{1}-b\right)$ form a theta, $K^{\prime}$. Since $V\left(K^{\prime}\right) \subseteq\left(V(K) \backslash V\left(S_{1}\right)\right) \cup\left(V(Q) \backslash\left\{s_{1}\right\}\right)$, and $|V(Q)| \leq\left|V\left(S_{1}\right)\right|$, we have $\left|V\left(K^{\prime}\right)\right|<|V(K)|$, contradicting the fact that no theta in $G$ is smaller than $K$. Therefore all neighbors of $q$ in $C$ are pairwise adjacent.

Suppose $q$ has exactly one neighbor in $C$; call it $u$. If $u$ is not adjacent to $b$ then the two paths between $u$ and $b$ in $C$ and a path with interior in $V\left(u-q-Q-m_{1}-T_{1}-b\right)$ form a theta, $K^{\prime}$. Since $V\left(K^{\prime}\right) \subseteq\left(V(K) \backslash V\left(S_{1}\right)\right) \cup(V(Q) \backslash$ $\left.\left\{s_{1}\right\}\right), K^{\prime}$ is smaller than $K$, a contradiction. Therefore $u$ is adjacent to $b$. Assume $u$ is not adjacent to $a$. Since $m_{1} \neq t_{1}$, the two paths between $u$ and $a$ in $C$ and a path $P^{\prime}$ between $u$ and $a$ with interior in $V\left(u-q-Q-m_{1}-S_{1}-s_{1}-a\right)$ form a theta, $K^{\prime}$. If $q$ is non-adjacent to $s_{1}$, then $\left|V\left(q-Q-m_{1}\right)\right|<\left|V\left(T_{1}\right)\right|$, and therefore $\left|V\left(K^{\prime}\right)\right|<|V(K)|$; and if $q$ is adajcent to $s_{1}$, then $V\left(P^{\prime}\right)=$ $\left\{q, s_{1}, a\right\}$, and so $V\left(K^{\prime}\right)=V(C) \cup\left\{s_{1}\right\}$ and, again, $\left|V\left(K^{\prime}\right)\right|<|V(K)|$, in both cases a contradiction. Therefore $u$ is adjacent to $a$. Since $u$ is adjacent to both $a$ and $b$, it is one of $m_{2}$ or $m_{3}$, contradicting the fact that $K$ has a tidy frame. Therefore, $q$ has two neighbors in $C$, and they are adjacent. This proves (1). 
Let $R$ be a bad shortcut across $T_{1}$. Suppose there is no edge between $V(R)$ and $V(C)$ except $t_{1}-b$. Since $R$ is a bad shortcut, it follows that some vertex of $R$ has a neighbor in $V\left(S_{1}\right) \backslash\left\{m_{1}\right\}$ and then the graph induced by $G$ on $V(C) \cup V(R) \cup V\left(S_{1}\right)$ contains a theta smaller than $K$, a contradiction. Since $m_{1}$ has no neighbors in $C$ and $b$ is the only neighbor of $t_{1}$ in $C$, this proves that some vertex in $R^{*}$ has a neighbor in $C$. Let $r \in R^{*}$ be the vertex with neighbors in $C$ such that no other vertex in $r-R-m_{1}$ has neighbors in C.

(2) The vertex $r$ has exactly two neighbors in $C$, and they are adjacent to each other.

This proof is very similar to that of (1). If $m_{1}=s_{1}$, then $m_{1}=t_{1}$ and there are no shortcuts between $m_{1}$ and $t_{1}$. Therefore we may assume $m_{1} \neq s_{1}$.

Suppose $r$ has two neighbors in $C$ that are not adjacent to each other. Let $u$ and $v$ be the neighbors of $r$ in $C$ such that a path between $u$ and $v$ in $C$ contains $a$ and no other neighbor of $r$. Then $u$ and $v$ are not adjacent to each other. Let $C_{u}$ be the path of $C$ from $u$ to $a$ such that $v \notin V\left(C_{u}\right)$ and let $C_{v}$ be the path of $C$ from $v$ to $a$ such that $u \notin V\left(C_{v}\right)$. The paths $r-u-C_{u}-a, r-v-C_{v^{-}} a$ and a path between $r$ and $a$ with interior contained in $V\left(r-R-m_{1}-S_{1}-a\right)$ form a theta, $K^{\prime}$. Since $V\left(K^{\prime}\right) \subseteq\left(V(K) \backslash V\left(T_{1}\right)\right) \cup\left(V(R) \backslash\left\{t_{1}\right\}\right)$, and $|V(R)| \leq\left|V\left(T_{1}\right)\right|$, we have $\left|V\left(K^{\prime}\right)\right|<|V(K)|$, contradicting the fact that no theta in $G$ is smaller than $K$. Therefore all neighbors of $r$ in $C$ are pairwise adjacent.

Suppose $r$ has exactly one neighbor in $C$; call it $u$. If $u$ is not adjacent to $a$ then the two paths between $u$ and $a$ in $C$ and a path with interior in $V\left(u-r-R-m_{1}-S_{1}-a\right)$ form a theta, $K^{\prime}$. Since $V\left(K^{\prime}\right) \subseteq\left(V(K) \backslash V\left(T_{1}\right)\right) \cup$ $\left(V(R) \backslash\left\{t_{1}\right\}\right), K^{\prime}$ is smaller than $K$, a contradiction. Therefore $u$ is adjacent to $a$. Assume $u$ is not adjacent to $b$. Since $m_{1} \neq s_{1}$, the two paths between $u$ and $b$ in $C$ and a path with interior in $V\left(u-r-R-m_{1}-T_{1}-t_{1}-b\right)$ form a theta, $K^{\prime}$. Since $r-R$ - $m_{1}$ contains fewer vertices than $R$ and $|V(R)| \leq\left|V\left(S_{1}\right)\right|$, we have $\left|V\left(K^{\prime}\right)\right|<|V(K)|$, a contradiction. Therefore $u$ is adjacent to $b$. Since $u$ is adjacent to both $a$ and $b$, it is one of $m_{2}$ or $m_{3}$, contradicting the fact that $K$ has a tidy frame. Therefore, $r$ has two neighbors in $C$, and they are adjacent. This proves (2).

Let $Q^{\prime}$ be another bad shortcut across $S_{1}$, and let $q^{\prime} \in Q^{\prime *}$ be the vertex with neighbors in $C$ such that no other vertex in $q^{\prime}-Q^{\prime}-m_{1}$ has neighbors in $C$. Denote by $x_{q}$ and $y_{q}$ the neighbors of $q$ in $C$, and by $x_{q}^{\prime}$ and $y_{q}^{\prime}$ the neighbors of $q^{\prime}$ in $C$. 
Let $R^{\prime}$ be another bad shortcut across $T_{1}$, and let $r^{\prime} \in R^{\prime *}$ be the vertex with neighbors in $C$ such that no other vertex in $r^{\prime}-R^{\prime}-m_{1}$ has neighbors in $C$. Denote by $x_{r}$ and $y_{r}$ the neighbors of $r$ in $C$, and by $x_{r}^{\prime}$ and $y_{r}^{\prime}$ the neighbors of $r^{\prime}$ in $C$.

(3) No two of the subsets $\left\{x_{q}, y_{q}\right\},\left\{x_{q}^{\prime}, y_{q}^{\prime}\right\},\left\{x_{r}, y_{r}\right\}$ and $\left\{x_{r}^{\prime}, y_{r}^{\prime}\right\}$ are disjoint.

Assume that $\left\{x_{q}, y_{q}\right\}$ is disjoint from $\left\{x_{q}^{\prime}, y_{q}^{\prime}\right\}$. Since $K$ has a tidy frame, $\{a, b\} \cap\left\{x_{q}, x_{q}^{\prime}, y_{q}, y_{q}^{\prime}\right\}=\emptyset$. Let $Q^{\prime \prime}$ be the path between $q$ and $q^{\prime}$ with interior in $V\left(q-Q-m_{1}\right) \cup V\left(q^{\prime}-Q^{\prime}-m_{1}\right)$. The triangles $\left\{q, x_{q}, y_{q}\right\}$ and $\left\{q^{\prime}, x_{q}^{\prime}, y_{q}^{\prime}\right\}$, the two paths between them in $C$ and the path $Q^{\prime \prime}$ form a prism, $K^{\prime}$. Since

$$
\left|V\left(Q^{\prime \prime}\right)\right| \leq 2\left|V\left(S_{1}\right)\right|-3<\left|V\left(S_{1}\right)\right|+\left|V\left(T_{1}\right)\right|-1,
$$

and $V\left(K^{\prime}\right) \subseteq V(C) \cup V\left(Q^{\prime \prime}\right)$, we deduce that $K^{\prime}$ is smaller than $K$, a contradiction. Therefore, $\left\{x_{q}, y_{q}\right\} \cap\left\{x_{q}^{\prime}, y_{q}^{\prime}\right\} \neq \emptyset$.

Assume that $\left\{x_{r}, y_{r}\right\}$ is disjoint from $\left\{x_{r}^{\prime}, y_{r}^{\prime}\right\}$. Since $K$ has a tidy frame, $\{a, b\} \cap\left\{x_{r}, x_{r}^{\prime}, y_{r}, y_{r}^{\prime}\right\}=\emptyset$. Let $R^{\prime \prime}$ be the path between $r$ and $r^{\prime}$ with interior in $V\left(r-R-m_{1}\right) \cup V\left(r^{\prime}-R^{\prime}-m_{1}\right)$. The triangles $\left\{r, x_{r}, y_{r}\right\}$ and $\left\{r^{\prime}, x_{r}^{\prime}, y_{r}^{\prime}\right\}$, the two paths between them in $C$ and the path $R^{\prime \prime}$ form a prism, $K^{\prime}$. Since

$$
\left|V\left(R^{\prime \prime}\right)\right| \leq 2\left|V\left(T_{1}\right)\right|-3<\left|V\left(S_{1}\right)\right|+\left|V\left(T_{1}\right)\right|-2,
$$

and $V\left(K^{\prime}\right) \subseteq V(C) \cup V\left(R^{\prime \prime}\right)$, we deduce that $K^{\prime}$ is smaller than $K$, a contradiction. Therefore, $\left\{x_{r}, y_{r}\right\} \cap\left\{x_{r}^{\prime}, y_{r}^{\prime}\right\} \neq \emptyset$.

Suppose $\left\{x_{q}, y_{q}\right\}$ is disjoint from $\left\{x_{r}, y_{r}\right\}$. Then the triangles $\left\{q, x_{q}, y_{q}\right\}$ and $\left\{r, x_{r}, y_{r}\right\}$, the two paths in $C$ between $\left\{x_{q}, y_{q}\right\}$ and $\left\{x_{r}, y_{r}\right\}$ and the path between $q$ and $r$ with interior in $V\left(q-Q-m_{1}-R-r\right)$ form a prism, $K^{\prime}$. Since $V\left(K^{\prime}\right) \subseteq V(C) \cup V(Q) \cup V(R) \backslash\left\{s_{1}, t_{1}\right\}$, the prism $K^{\prime}$ is smaller than $K$, a contradiction. Therefore $\left\{x_{q}, y_{q}\right\} \cap\left\{x_{r}, y_{r}\right\} \neq \emptyset$. By symmetry, we also have that $\left\{x_{q}, y_{q}\right\} \cap\left\{x_{r}^{\prime}, y_{r}^{\prime}\right\} \neq \emptyset,\left\{x_{q}^{\prime}, y_{q}^{\prime}\right\} \cap\left\{x_{r}, y_{r}\right\} \neq \emptyset$, and $\left\{x_{q}^{\prime}, y_{q}^{\prime}\right\} \cap\left\{x_{r}^{\prime}, y_{r}^{\prime}\right\} \neq \emptyset$. This proves (3).

Let $U, W$ and $Z$ each be a bad shortcut across either $S_{1}$ or $T_{1}$. Since the choices of $Q, Q^{\prime}, R$ and $R^{\prime}$ were arbitrary, it follows from (1), (2) and (3) that there exist $u \in U^{*}, w \in W^{*}$ and $z \in Z^{*}$ such that $u$ has two adjacent neighbors $x_{u}, y_{u} \in V(C), w$ has two adjacent neighbors $x_{w}, y_{w} \in V(C), z$ has two adjacent neighbors $x_{z}, y_{z} \in V(C)$, and no two of $\left\{x_{u}, y_{u}\right\},\left\{x_{w}, y_{w}\right\}$ and $\left\{x_{z}, y_{z}\right\}$ are disjoint.

Assume that $\left\{x_{w}, y_{w}\right\} \cap\left\{x_{z}, y_{z}\right\}=\left\{x_{w}\right\}$ and $\left\{x_{w}, y_{w}\right\} \cap\left\{x_{u}, y_{u}\right\}=\left\{y_{w}\right\}$. Then, since $x_{w}$ and $y_{w}$ are adjacent and $x_{z}$ and $y_{z}$ are adjacent, $\left\{x_{z}, y_{z}\right\}$ 
contains $x_{w}$ and the vertex adjacent to $x_{w}$ in $V(C) \backslash\left\{y_{w}\right\}$. Similarly, since $x_{u}$ and $y_{u}$ are adjacent, $\left\{x_{u}, y_{u}\right\}$ contains $y_{w}$ and the vertex adjacent to $y_{w}$ in $V(C) \backslash\left\{x_{w}\right\}$. Since $x_{w}$ and $y_{w}$ have no common neighbors in $C$, we have that $\left\{x_{z}, y_{z}\right\} \cap\left\{x_{u}, y_{u}\right\}=\emptyset$, a contradiction. Therefore, by symmetry between $x_{w}$ and $y_{w}$ we may assume that $x_{w} \in\left\{x_{z}, y_{z}\right\}$ and $x_{w} \in\left\{x_{u}, y_{u}\right\}$. Therefore every bad shortcut across $S_{1}$ or $T_{1}$ contains an interior vertex adjacent to $x_{w}$; taking $c=x_{w}$ proves 2.1 .

2.2. There is an algorithm with the following specifications:

- Input: A graph $G$.

- Output: $A$ sequence of subsets $X_{1}, \ldots, X_{r}$ of $V(G)$ with $r \leq|V(G)|^{3}$, such that for every smallest theta $K$ in $G$ with a tidy frame and $i \in$ $\{1,2,3\}$, one of $X_{1}, \ldots, X_{r}$ is disjoint from $V(K)$ and contains a vertex of every bad shortcut across the half-paths $S_{i}$ and $T_{i}$ of $K$.

- Running Time: $O\left(|V(G)|^{4}\right)$.

Proof. The algorithm is as follows. Enumerate all triples of vertices $(c, u, v)$ for which $u$ and $v$ are adjacent to $c$. For each such triple, compute the subset $N(c) \backslash\{u, v\}$. Let $X_{1}, \ldots, X_{r}$ be the subsets generated. We output the list $\emptyset, X_{1}, \ldots, X_{r}$. That concludes the description of the algorithm; we now prove that it works correctly. The number of subsets generated is at most $|V(G)|^{3}$ and the running time is $O\left(|V(G)|^{4}\right)$ because it takes linear time to compute $N(c)$. It remains to check that the sequence $X_{1}, \ldots, X_{r}$ has the properties claimed. Let $K$ be a smallest theta in $G$ and assume it has a tidy frame. By 2.1, for every $i=1,2,3$ there is a vertex $c^{\prime} \in V(K)$ such that every bad shortcut across $S_{i}$ or $T_{i}$ contains an interior vertex adjacent to $c^{\prime}$. The vertex $c^{\prime}$ has exactly two neighbors in $K$; call them $u^{\prime}$ and $v^{\prime}$. One of the triples enumerated by the algorithm will be $\left(c^{\prime}, u^{\prime}, v^{\prime}\right)$. The subset $N\left(c^{\prime}\right) \backslash\left\{u^{\prime}, v^{\prime}\right\}$ includes all neighbors of $c^{\prime}$ except its two neighbors that are in $K$, and is therefore disjoint from $V(K)$. This proves 2.2 .

\section{Prism shortcuts}

Let $K$ be a prism in $G$. Label the triangles of $K\left\{a_{1}, a_{2}, a_{3}\right\}$ and $\left\{b_{1}, b_{2}, b_{3}\right\}$ and the paths $P_{1}, P_{2}$ and $P_{3}$ such that for $i=1,2,3$ the path $P_{i}$ has ends $a_{i}$ and $b_{i}$. For $i=1,2,3$ choose $m_{i} \in P_{i}^{*}$ and subpaths $S_{i}$ and $T_{i}$

such that $S_{i}=a_{i}-P_{i}-m_{i}, T_{i}=m_{i}-P_{i}-b_{i}$ and $\left|V\left(S_{i}\right)\right| \leq\left|V\left(T_{i}\right)\right|+1$ and $\left|V\left(T_{i}\right)\right| \leq\left|V\left(S_{i}\right)\right|+1$. We call $P_{1}, P_{2}$ and $P_{3}$ the paths of $K$ and $S_{1}, T_{1}, S_{2}$, 
$T_{2}, S_{3}$, and $T_{3}$ the half-paths of $K$. The 9-tuple

$$
\left\{a_{1}, a_{2}, a_{3}, m_{1}, m_{2}, m_{3}, b_{1}, b_{2}, b_{3}\right\}
$$

is the frame of $K$. A prism $K$ has a tidy frame if for $i=1,2,3$,

- neither $a_{i}$ nor $b_{i}$ has neighbors in $V(G) \backslash V(K)$, and

- if $m_{i}$ is adjacent to $a_{i}$ and $b_{i}$, then $m_{i}$ has no neighbors in $V(G) \backslash V(K)$.

The prism $K$ in $G$ is almost clean if it has a tidy frame and $G$ contains no $K$-major vertices.

If $S$ is one of the half-paths of $K$, then a shortcut across $S$ is a shortest path in $G$ with the same ends as $S$. A shortcut across $K$ is a shortcut across some half-path of $K$. A shortcut $Q$ across $S$ is good if no interior vertex of $Q$ has neighbors in $V(K) \backslash V(S)$, and bad otherwise.

Let $C$ be the cycle induced by $G$ on $V\left(P_{2}\right) \cup V\left(P_{3}\right)$.

3.1. Let $K$ be a smallest prism. For every bad shortcut $Q$ across $S_{1}$, some vertex of $Q^{*}$ has a neighbor in $C$.

Proof. Suppose no vertex of $Q^{*}$ has a neighbor in $C$. Then, since $Q$ is bad, some vertex of $Q^{*}$ has a neighbor in $V\left(T_{1}\right) \backslash\left\{m_{1}\right\}$. Therefore, there exists a path $P_{1}^{\prime}$ from $a_{1}$ to $b_{1}$ with $P_{1}^{\prime *} \subset V(Q) \cup V\left(T_{1}\right) \backslash\left\{m_{1}\right\}$. So there is a prism contained in $V(C) \cup V\left(P_{1}^{\prime}\right)$ that is smaller than $K$, a contradiction because $K$ is a smallest prism. This proves 3.1.

In view of 3.1 , for a bad shortcut $Q$ across $S_{1}$ or $T_{1}$, define $\beta_{Q} \in Q^{*}$ to be the vertex with neighbors in $V(C)$ such that no other vertex in $\beta_{Q}-Q-m_{1}$ has neighbors in $V(C)$.

3.2. Let $K$ be an almost clean smallest prism. For a bad shortcut $Q$ across $S_{1}$, either $\left|N\left(\beta_{Q}\right) \cap V(K)\right|=\left|N\left(\beta_{Q}\right) \cap V(C)\right|=1$ or $N\left(\beta_{Q}\right) \cap V(K)$ is the vertex set of a 3-vertex path of $C$.

Proof. Since $K$ is almost clean, $\beta_{Q}$ is not a $K$-major vertex, and therefore $N\left(\beta_{Q}\right) \cap V(K) \subseteq N\left(\beta_{Q}\right) \cap V(C)$. Assume $\beta_{Q}$ has exactly two neighbors in $C, u$ and $v$. If $u$ and $v$ are not adjacent, then $u-\beta_{Q}-v$ and the two paths in $C$ between $u$ and $v$ form a theta that has fewer vertices than $K$, a contradiction. So $u$ and $v$ are adjacent. Denote $\beta_{Q}-Q-m_{1}$ by $U$. Since $K$ is almost clean, $\{u, v\} \cap\left\{b_{1}, b_{2}, b_{3}\right\}=\emptyset$, so there is a prism $K^{\prime}$ containing the triangles $\left\{b_{1}, b_{2}, b_{3}\right\}$ and $\left\{\beta_{Q}, u, v\right\}$ with $V\left(K^{\prime}\right) \subseteq V(C) \cup V\left(T_{1}\right) \cup V(U)$ (the three paths of $K^{\prime}$ are two paths of $C$ and the path contained in $V(U) \cup V\left(T_{1}\right)$ ). Since $|V(U)|<\left|V\left(S_{1}\right)\right|$ (because $a_{1} \notin V(U)$ ), $K^{\prime}$ is smaller than $K$, a 
contradiction. Therefore, $\beta_{Q}$ has either a unique neighbor in $C$ or at least three neighbors in $C$. Since $K$ is almost clean, $\beta_{Q}$ is not $K$-major, so if it has at least three neighbors in $C$ then it has exactly three neighbors in $C$, which are the vertex set of a 3 -vertex path of $C$. This proves 3.2.

3.3. Let $K$ be a smallest prism in $G$. Assume $K$ is almost clean. Then there exist two edges $e_{1}$ and $e_{2}$, one in $E\left(P_{2}\right)$ and the other in $E\left(P_{3}\right)$, such that either

- there exists a bad shortcut $T$ across $S_{1}$ with $\left|N\left(\beta_{T}\right) \cap V(C)\right|=1$ and for every bad shortcut $Q$ across $S_{1}$, if $\left|N\left(\beta_{Q}\right) \cap V(C)\right|=1$ then $\beta_{Q}$ is adjacent to an end of $e_{1}$ and if $\left|N\left(\beta_{Q}\right) \cap V(C)\right|>1$ then $\beta_{Q}$ is adjacent to both ends of $e_{1}$, or

- for every bad shortcut $Q,\left|N\left(\beta_{Q}\right) \cap V(C)\right|=3$ and $\beta_{Q}$ is adjacent either to both ends of $e_{1}$ or to both ends of $e_{2}$.

Proof. We start with a few observations about bad shortcuts across $S_{1}$.

(1) There exists an edge $e_{1} \in E(C)$ such that for every bad shortcut $Q$ across $S_{1}$, if $\left|N\left(\beta_{Q}\right) \cap V(C)\right|=1$ then $\beta_{Q}$ is adjacent to an end of $e_{1}$.

We may assume that there exist two distinct bad shortcuts $Q$ and $Q^{\prime}$ across $S_{1}$ such that $\beta_{Q}$ has a unique neighbor $x$ in $C, \beta_{Q^{\prime}}$ has a unique neighbor $y$ in $C$, and $x \neq y$. Let $U$ be the path $\beta_{Q^{-}} Q-m_{1}$, let $U^{\prime}$ be the path $\beta_{Q^{\prime}} Q^{\prime}-m_{1}$ and let $W$ be a path between $\beta_{Q}$ and $\beta_{Q^{\prime}}$ with interior in $V(U) \cup$ $V\left(U^{\prime}\right)$. If $x$ and $y$ are nonadjacent, then the two paths between them in $C$ and the path $x-\beta_{Q^{-}} W-\beta_{Q^{\prime}} y$ form a theta, $K^{\prime}$. Since $|V(W)| \leq 2\left|V\left(S_{1}\right)\right|-3$ and $\left|V\left(P_{1}\right)\right| \geq 2\left|V\left(S_{1}\right)\right|-2, K^{\prime}$ is smaller than $K$, a contradiction. Therefore, $x$ is adjacent to $y$.

Let $Q^{\prime \prime}$ be any other bad shortcut across $S_{1}$ such that $\beta_{Q^{\prime \prime}}$ has a unique neighbor $z$ in $C$. By the previous argument, applied to the pairs $Q, Q^{\prime \prime}$ and $Q^{\prime}, Q^{\prime \prime}$, it follows that $z$ is equal or adjacent to $x$ and equal or adjacent to $y$. Then either $z \in\{x, y\}$ or $z$ is adjacent to both $x$ and $y$. But since $x$ and $y$ are adjacent vertices of $C$, they have no common neighbor in $C$, so $z \in\{x, y\}$. Therefore, there exists an edge $e_{1}=x-y$ such that whenever $\beta_{Q^{\prime \prime}}$ has a unique neighbor in $C, \beta_{Q^{\prime \prime}}$ is adjacent to an end of $e_{1}$. This proves (1).

(2) There exist edges $e_{1} \in E\left(P_{2}\right)$ and $e_{2} \in E\left(P_{3}\right)$ such that for every bad shortcut $Q$ across $S_{1}$, if $\left|N\left(\beta_{Q}\right) \cap V(C)\right|=3$ then $\beta_{Q}$ is either adjacent to both ends of $e_{1}$ or to both ends of $e_{2}$. 
Let $Q$ be a bad shortcut across $S_{1}$ such that $\beta_{Q^{\prime}}$ has three neighbors in $C$. By 3.2, $N\left(\beta_{Q}\right) \cap V(C)$ is the vertex set of a 3 -vertex path of $C$. Since $K$ is almost clean, $N\left(\beta_{Q}\right) \cap V(C)$ is contained in either $P_{2}^{*}$ or $P_{3}^{*}$.

Therefore, in order to prove (2), it is enough to show that there exist edges $e_{1} \in E\left(P_{2}\right)$ and $e_{2} \in E\left(P_{3}\right)$ such that for every bad shortcut $Q$ across $S_{1}$, if $\left|N\left(\beta_{Q}\right) \cap V\left(P_{2}\right)\right|=3$ then $\beta_{Q}$ is adjacent to both ends of $e_{1}$, and if $\left|N\left(\beta_{Q}\right) \cap V\left(P_{3}\right)\right|=3$, then $\beta_{Q}$ is adjacent to both ends of $e_{2}$.

Let $Q$ and $Q^{\prime}$ be bad shortcuts across $S_{1}$. Suppose that each of $\beta_{Q}$ and $\beta_{Q^{\prime}}$ has three neighbors in $P_{2}$. Then each of $N\left(\beta_{Q}\right) \cap V(C)$ and $N\left(\beta_{Q^{\prime}}\right) \cap$ $V(C)$ is the vertex set of a 3 -vertex path of $P_{2}$.

We claim that $\left|N\left(\beta_{Q}\right) \cap V(C) \cap N\left(\beta_{Q^{\prime}}\right)\right| \geq 2$. Assume that $\mid N\left(\beta_{Q}\right) \cap$ $V(C) \cap N\left(\beta_{Q^{\prime}}\right) \mid<2$. If $\beta_{Q}$ and $\beta_{Q^{\prime}}$ are adjacent to each other, then there is a path $P_{2}^{\prime}$ between $a_{2}$ and $b_{2}$ whose interior is contained in $P_{2}^{*} \cup\left\{\beta_{Q}, \beta_{Q^{\prime}}\right\}$ and is anticomplete to $V(K) \backslash P_{2}^{*}$. Then $\left(V(K) \backslash P_{2}^{*}\right) \cup P_{2}^{\prime *}$ contains a prism smaller than $K$, a contradiction. So we may assume that $\beta_{Q}$ and $\beta_{Q^{\prime}}$ are not adjacent to each other. Then there are two paths $A$ and $B$ between $\beta_{Q}$ and $\beta_{Q^{\prime}}$ whose interiors are anticomplete to each other and are contained in $V(C)$. The paths $A, B$ and a path between $\beta_{Q}$ and $\beta_{Q^{\prime}}$ with interior in $V(Q) \cup V\left(Q^{\prime}\right)$ form a theta, $K^{\prime}$. Since $\left|V(Q) \cup V\left(Q^{\prime}\right)\right| \leq 2\left|V\left(S_{1}\right)\right|-3<$ $\left|V\left(S_{1}\right)\right|+\left|V\left(T_{1}\right)\right|-1=\left|V\left(P_{1}\right)\right|$, the theta $K^{\prime}$ is smaller than the prism $K$, a contradiction. This proves the claim.

Label the neighbors of $\beta_{Q}$ in $C x, y$ and $z$ and the neighbors of $\beta_{Q^{\prime}}$ in $C$ $x^{\prime}, y^{\prime}$ and $z^{\prime}$ such that $x-y-z$ and $x^{\prime}-y^{\prime}-z^{\prime}$ are paths of $C$. Let $Q^{\prime \prime}$ be another bad shortcut across $S_{1}$ such that $\beta_{Q^{\prime \prime}}$ has three neighbors $x^{\prime \prime}, y^{\prime \prime}$ and $z^{\prime \prime}$ in $P_{2}$ and $x^{\prime \prime}-y^{\prime \prime}-z^{\prime \prime}$ is a path of $P_{2}$. Then we have that $\left|\left\{x^{\prime \prime}, y^{\prime \prime}, z^{\prime \prime}\right\} \cap\{x, y, z\}\right| \geq 2$ and $\left|\left\{x^{\prime \prime}, y^{\prime \prime}, z^{\prime \prime}\right\} \cap\left\{x^{\prime}, y^{\prime}, z^{\prime}\right\}\right| \geq 2$. Since $x-y-z$ is a path, either $\{x, y\} \subset$ $\left\{x^{\prime}, y^{\prime}, z^{\prime}\right\}$ or $\{y, z\} \subset\left\{x^{\prime}, y^{\prime}, z^{\prime}\right\}$, and similarly, either $\{x, y\} \subset\left\{x^{\prime \prime}, y^{\prime \prime}, z^{\prime \prime}\right\}$ or $\{y, z\} \subset\left\{x^{\prime \prime}, y^{\prime \prime}, z^{\prime \prime}\right\}$. Assume that $\{x, y, z\} \cap\left\{x^{\prime}, y^{\prime}, z^{\prime}\right\}=\{x, y\}$ and $\{x, y, z\} \cap\left\{x^{\prime \prime}, y^{\prime \prime}, z^{\prime \prime}\right\}=\{y, z\}$. Then since $x-y-z$ is a path and $x^{\prime}-y^{\prime}-z^{\prime}$ is a path, $\left\{x^{\prime}, y^{\prime}, z^{\prime}\right\}$ contains $\{x, y\}$ and the vertex adjacent to $x$ in $V\left(P_{2}\right) \backslash\{y\}$. Similarly, since $x^{\prime \prime}-y^{\prime \prime}-z^{\prime \prime}$ is a path, $\left\{x^{\prime \prime}, y^{\prime \prime}, z^{\prime \prime}\right\}$ contains $\{y, z\}$ and the vertex adjacent to $z$ in $V\left(P_{2}\right) \backslash\{y\}$. Since $x$ and $z$ are not adjacent and $x$ and $z$ have no common neighbors in $V\left(P_{2}\right) \backslash\{y\}$, it follows that $\left\{x^{\prime}, y^{\prime}, z^{\prime}\right\} \cap\left\{x^{\prime \prime}, y^{\prime \prime}, z^{\prime \prime}\right\}=$ $\{y\}$, a contradiction. Therefore, by symmetry between $\{x, y\}$ and $\{y, z\}$ we may assume that $\{x, y\} \subset\left\{x^{\prime}, y^{\prime}, z^{\prime}\right\}$ and $\{x, y\} \subset\left\{x^{\prime \prime}, y^{\prime \prime}, z^{\prime \prime}\right\}$. This proves that for every bad shortcut $R$ across $S_{1}$, if $\left|N\left(\beta_{R}\right) \cap V\left(P_{2}\right)\right|=3$ then $\beta_{R}$ is adjacent to both ends of $e_{1}=x-y$.

From the symmetry, there exists an edge $e_{2} \in E\left(P_{3}\right)$ such that for every bad shortcut $R$ across $S_{1}$, if $\left|N\left(\beta_{R}\right) \cap V\left(P_{3}\right)\right|=3$ then $\beta_{R}$ is adjacent to both ends of $e_{2}$. This proves $(2)$. 
(3) If $Q$ and $Q^{\prime}$ are bad shortcuts across $S_{1}$ with $\left|N\left(\beta_{Q}\right) \cap V(C)\right|=1$ and $\left|N\left(\beta_{Q^{\prime}}\right) \cap V(C)\right|=3$, then the neighbor of $\beta_{Q}$ in $C$ is adjacent to $\beta_{Q^{\prime}}$.

Denote by $x$ the neighbor of $\beta_{Q}$ in $C$. By 3.2, the neighbors of $\beta_{Q^{\prime}}$ in $C$ are the vertex set of a 3 -vertex path of $C$, say $c_{1}-c_{2}-c_{3}$.

Assume that $x \notin\left\{c_{1}, c_{2}, c_{3}\right\}$. Then the subgraph induced by $G$ on $\left(V(C) \backslash\left\{c_{2}\right\}\right) \cup V(Q) \cup V\left(Q^{\prime}\right)$ contains a theta, $K^{\prime}$ (the three paths of $K^{\prime}$ are two paths between $\beta_{Q^{\prime}}$ and $x$ with interior in $C$ and the path with interior in $\left.V\left(\beta_{Q^{\prime}}-Q^{\prime}-m_{1}-Q-\beta_{Q^{-}}-x\right)\right)$. Since $V\left(K^{\prime}\right) \subseteq V(C) \cup Q^{*} \cup Q^{\prime *} \cup\left\{m_{1}\right\}$ and $\left|Q^{*}\right|+\left|Q^{\prime *}\right|+1 \leq 2\left|V\left(S_{1}\right)\right|-3<\left|V\left(S_{1}\right)\right|+\left|V\left(T_{1}\right)\right|-1$, the theta $K^{\prime}$ is smaller than $K$, a contradiction. Therefore, $x \in\left\{c_{1}, c_{2}, c_{3}\right\}$. This proves $(3)$.

If for every bad shortcut $Q,\left|N\left(\beta_{Q}\right) \cap V(C)\right|=3$, then the second outcome of the theorem holds by (2). So we may assume that there exists a bad shortcut $Q$ with $N\left(\beta_{Q}\right) \cap V(C)=\{x\}$. Suppose there exists a bad shortcut $Q^{\prime}$ with $\left|N\left(\beta_{Q^{\prime}}\right) \cap V(C)\right|=\{y\}$ such that $x \neq y$. Then by (1) and (3) the first outcome of the theorem holds with $e_{1}$ being the edge as in (1). So we may assume that for every bad shortcut $Q^{\prime}$ with $\left|N\left(\beta_{Q^{\prime}}\right) \cap V(C)\right|=1, \beta_{Q^{\prime}}$ is adjacent to $x$. Let $c_{1}-x-c_{2}$ be the 3 -vertex path in $C$ with $x$ as its interior vertex. If there exist bad shortcuts $Q^{\prime}$ and $Q^{\prime \prime}$ with $\left|N\left(\beta_{Q^{\prime}}\right) \cap V(C)\right|=3$ and $\left|N\left(\beta_{Q^{\prime \prime}}\right) \cap V(C)\right|=3$ such that $\beta_{Q^{\prime}}$ is not adjacent to $c_{1}$ and $\beta_{Q^{\prime \prime}}$ is not adjacent to $c_{2}$, then $N\left(\beta_{Q^{\prime}}\right) \cap V(C) \cap N\left(\beta_{Q^{\prime \prime}}\right)=\{x\}$, contradicting (2). Thus one of $c_{1}$ and $c_{2}$, say $c_{1}$, is complete to $\left\{\beta_{Q^{\prime}}, \beta_{Q^{\prime \prime}}\right\}$, and the theorem holds with $e_{1}=c_{1}-x$. This completes the proof of 3.3.

3.4. Let $K$ be a smallest prism in $G$. Assume $K$ is almost clean. Then there exist two edges $e_{1}$ and $e_{2}$, one in $E\left(P_{2}\right)$ and the other in $E\left(P_{3}\right)$, such that either

- there exists a bad shortcut $T$ across $S_{1}$ or $T_{1}$ with $\left|N\left(\beta_{T}\right) \cap V(C)\right|=1$, and for every bad shortcut $Q$ across $S_{1}$ or $T_{1}$, if $\left|N\left(\beta_{Q}\right) \cap V(C)\right|=1$ then $\beta_{Q}$ is adjacent to an end of $e_{1}$ and if $\left|N\left(\beta_{Q}\right) \cap V(C)\right|>1$ then $\beta_{Q}$ is adjacent to both ends of $e_{1}$, or

- for every bad shortcut $Q$ across $S_{1}$ or $T_{1},\left|N\left(\beta_{Q}\right) \cap V(C)\right|=3$ and $\beta_{Q}$ is adjacent either to both ends of $e_{1}$ or to both ends of $e_{2}$.

Proof. We start with a few observations about bad shortcuts across $S_{1}$ and $T_{1}$. 
(1) Let $Q$ be a bad shortcut across $S_{1}$ with $\left|N\left(\beta_{Q}\right) \cap V(C)\right|=1$ and $R$ a bad shortcut across $T_{1}$ with $\left|N\left(\beta_{R}\right) \cap V(C)\right|=1$. Then the neighbor of $\beta_{Q}$ in $C$ and the neighbor of $\beta_{R}$ in $C$ are either equal or adjacent to each other.

Let $\left\{x_{q}\right\}=N\left(\beta_{Q}\right) \cap V(C)$ and $\left\{x_{r}\right\}=N\left(\beta_{R}\right) \cap V(C)$. Assume that $x_{q}$ and $x_{r}$ are distinct and nonadjacent. There are two paths between them, $A$ and $B$, each of length at least two and contained in $C$. There is a path $P$ between $x_{q}$ and $x_{r}$ with interior in $V\left(\beta_{Q}-Q-m_{1}-R-\beta_{R}\right)$. The three paths $A$, $B$ and $P$ form a theta, $K^{\prime}$. Since $|V(P)| \leq\left|Q^{*} \cup\left\{m_{1}\right\} \cup R^{*}\right|<\left|V\left(P_{1}\right)\right|$, the theta $K^{\prime}$ is smaller than the prism $K$, a contradiction. This proves (1).

(2) Let $Q$ be a bad shortcut across $S_{1}$ with $\left|N\left(\beta_{Q}\right) \cap V(C)\right|=3$ and $N\left(\beta_{Q}\right) \cap$ $V(C) \subseteq P_{2}^{*}$. Let $R$ be a bad shortcut across $T_{1}$ with $\left|N\left(\beta_{R}\right) \cap V(C)\right|=3$ and $N\left(\beta_{R}\right) \cap V(C) \subseteq P_{2}^{*}$. Then there is an edge in $E\left(P_{2}\right)$ whose ends are $\left\{\beta_{Q}, \beta_{R}\right\}$-complete.

Assume that $\left|N\left(\beta_{Q}\right) \cap V(C) \cap N\left(\beta_{R}\right)\right|<2$. By 3.2, each of $N\left(\beta_{Q}\right) \cap V(K)$ and $N\left(\beta_{R}\right) \cap V(K)$ is the vertex set of a 3 -vertex path of $C$. If $\beta_{Q}$ is adjacent to $\beta_{R}$ then there is a path $A$ between $a_{2}$ and $b_{2}$ with interior in $P_{2}^{*} \cup\left\{\beta_{Q}, \beta_{R}\right\}$ such that $\left\{\beta_{Q}, \beta_{R}\right\} \subset V(A)$. Since $\left|N\left(\beta_{Q}\right) \cap V(C)\right|=\left|N\left(\beta_{R}\right) \cap V(C)\right|=3$, it follows that $\left|\left(N\left(\beta_{Q}\right) \cup N\left(\beta_{R}\right)\right) \cap V(C)\right| \geq 5$. So $\left|V\left(P_{2}\right) \backslash V(A)\right| \geq 3$ and $\left|V(A) \backslash V\left(P_{2}\right)\right|=2$, which implies that $|V(A)|<\left|V\left(P_{2}\right)\right|$. It follows from 3.2 that $A^{*}$ is anticomplete to $P_{1}^{*}$ and $P_{3}^{*}$, and so the induced subgraph with vertex set $\left(V(K) \backslash V\left(P_{2}\right)\right) \cup V(A)$ is a prism smaller than $K$, a contradiction. This proves that $\beta_{Q}$ is not adjacent to $\beta_{R}$. It follows that there are two paths $B$ and $B^{\prime}$ between $\beta_{Q}$ and $\beta_{R}$ whose interiors are anticomplete to each other and are contained in $V(C)$. There is also a path $P$ between them with interior contained in $V\left(\beta_{Q}-Q-m_{1}-R-\beta_{R}\right)$. Since $\beta_{Q}$ is not adjacent to $\beta_{R}$, $P$ has length at least two. Since $|V(P)| \leq\left|Q^{*} \cup\left\{m_{1}\right\} \cup R^{*}\right|<\left|V\left(P_{1}\right)\right|$, the paths $B, B^{\prime}$ and $P$ form a theta smaller than $K$, a contradiction. This proves that $\left|N\left(\beta_{Q}\right) \cap V(C) \cap N\left(\beta_{R}\right)\right| \geq 2$. Since each of $N\left(\beta_{Q}\right) \cap V(C)$ and $N\left(\beta_{R}\right) \cap V(C)$ is the vertex set of a 3 -vertex subpath of $P_{2}$, (2) follows.

(3) Let $Q$ be a bad shortcut across $S_{1}$ with $\left|N\left(\beta_{Q}\right) \cap V(C)\right|=1$ and $R$ a bad shortcut across $T_{1}$ with $\left|N\left(\beta_{R}\right) \cap V(C)\right|=3$. Then the neighbor of $\beta_{Q}$ in $C$ is adjacent to $\beta_{R}$.

Let $\left\{x_{q}\right\}=N\left(\beta_{Q}\right) \cap V(C)$. Assume that $x_{q} \notin N\left(\beta_{R}\right)$. There are two paths $A$ and $B$ between $x_{q}$ and $\beta_{R}$ whose interiors are anticomplete to each other and are contained in $V(C)$. There is a path $P$ between $x_{q}$ and $\beta_{R}$ with interior in $V\left(\beta_{Q^{-}} Q-m_{1}-R-\beta_{R}\right)$. Since $|V(P)| \leq\left|Q^{*} \cup\left\{m_{1}\right\} \cup R^{*}\right|+1<\left|V\left(P_{1}\right)\right|$, the paths $A, B$ and $P$ form a theta smaller than $K$, a contradiction. This proves (3). 
(4) Suppose that there exist $p \in V\left(P_{2}\right)$ and $s \in V\left(P_{3}\right)$ such that for every bad shortcut $Q$ across $S_{1}$ or $T_{1}, \beta_{Q}$ is adjacent to $p$ or $s$. Then the theorem holds.

Since there are no $K$-major vertices in $G$, for every bad shortcut $Q$ across $S_{1}$ or $T_{1}$ with $N\left(\beta_{Q}\right) \cap V(C) \subseteq P_{2}^{*}, N\left(\beta_{Q}\right) \cap V(C)$ is contained in the vertex set of the 5 -vertex path $c_{1}-c_{2}-p-c_{3}-c_{4}$ contained in $C$. Suppose there exist two bad shortcuts $P$ and $P^{\prime}$ across either $S_{1}$ or $T_{1}$ with $N\left(\beta_{P}\right) \cap V(C)=$ $\left\{c_{1}, c_{2}, p\right\}$ and $N\left(\beta_{P^{\prime}}\right) \cap V(C)=\left\{p, c_{3}, c_{4}\right\}$. Then $N\left(\beta_{P}\right) \cap V(C) \cap N\left(\beta_{P^{\prime}}\right)=$ $\{p\}$. If both $P$ and $P^{\prime}$ are shortcuts across $S_{1}$ or both are shortcuts across $T_{1}$, then this contradicts both outcomes of 3.3. If one of $P$ and $P^{\prime}$ is a shortcut across $S_{1}$ and the other is a shortcut across $T_{1}$, then this contradicts (2). Therefore, there exists a 4 -vertex path $A$ of $C$ with $p \in A^{*}$ such that for every bad shortcut $P$ across $S_{1}$ or $T_{1}$, if $N\left(\beta_{P}\right) \cap V(C) \subseteq P_{2}^{*}$ then $N\left(\beta_{P}\right) \cap V(C) \subset$ $V(A)$. Let $e_{1}$ be the edge with both ends in $A^{*}$. It follows that for every bad shortcut $P$ across $S_{1}$ or $T_{1}$ with $N\left(\beta_{P}\right) \cap V(C) \subseteq P_{2}^{*}$, if $\left|N\left(\beta_{P}\right) \cap V(C)\right|=1$ then $\beta_{P}$ is adjacent to the end $p$ of $e_{1}$, and if $\left|N\left(\beta_{P}\right) \cap V(C)\right|>1$ then $\beta_{P}$ is adjacent to both ends of $e_{1}$. By symmetry between $P_{2}$ and $P_{3}$, it also follows that there is an edge $e_{2} \in E\left(P_{3}\right)$ such that for every bad shortcut $P$ across $S_{1}$ or $T_{1}$ with $N\left(\beta_{P}\right) \cap V(C) \subseteq P_{3}^{*}$, if $\left|N\left(\beta_{P}\right) \cap V(C)\right|=1$ then $\beta_{P}$ is adjacent to an end of $e_{2}$, and if $\left|N\left(\beta_{P}\right) \cap V(C)\right|>1$ then $\beta_{P}$ is adjacent to both ends of $e_{2}$. So if for all bad shortcuts $Q$ across $S_{1}$ and $T_{1},\left|N\left(\beta_{Q}\right) \cap V(C)\right|>1$, then the second outcome of the theorem holds. If not, then by (1) and (3), either for all bad shortcuts $Q$ across $S_{1}$ and $T_{1}, N\left(\beta_{Q}\right) \cap V(C) \subseteq P_{2}^{*}$, or for all bad shortcuts $Q$ across $S_{1}$ and $T_{1}, N\left(\beta_{Q}\right) \cap V(C) \subseteq P_{3}^{*}$. In both cases, (with $e_{2}$ in place of $e_{1}$ in the second case), the first outcome of the theorem holds. This proves (4).

First assume that there exist a bad shortcut $Q$ across $S_{1}$ with $\mid N\left(\beta_{Q}\right) \cap$ $V(C) \mid=1$ and a bad shortcut $R$ across $T_{1}$ with $\left|N\left(\beta_{R}\right) \cap V(C)\right|=1$. The first outcome of 3.3 holds for both $S_{1}$ and $T_{1}$. Let $\left\{x_{q}\right\}=N\left(\beta_{Q}\right) \cap V(C)$ and $\left\{x_{r}\right\}=N\left(\beta_{R}\right) \cap V(C)$. Assume that $x_{q} \neq x_{r}$. By (1), $x_{q}$ is adjacent to $x_{r}$. Let $Q^{\prime}$ be any other bad shortcut across $S_{1}$ and let $R^{\prime}$ be any other bad shortcut across $T_{1}$. It follows from (1) that if $\left|N\left(\beta_{Q^{\prime}}\right) \cap V(C)\right|=1$ then $\beta_{Q^{\prime}}$ is adjacent to $x_{q}$ or $x_{r}$; it also follows that if $\left|N\left(\beta_{R^{\prime}}\right) \cap V(C)\right|=1$ then $\beta_{R^{\prime}}$ is adjacent to $x_{r}$ or $x_{q}$. Also, 3.3 implies that if $\left|N\left(\beta_{Q^{\prime}}\right) \cap V(C)\right|>1$ then $\beta_{Q^{\prime}}$ is adjacent to $x_{q}$, and if $\left|N\left(\beta_{R^{\prime}}\right) \cap V(C)\right|>1$ then $\beta_{R^{\prime}}$ is adjacent to $x_{r}$. By (3), if $\left|N\left(\beta_{Q^{\prime}}\right) \cap V(C)\right|>1$ then $\beta_{Q^{\prime}}$ is adjacent to $x_{r}$ and if $\left|N\left(\beta_{R^{\prime}}\right) \cap V(C)\right|>1$ then $\beta_{R^{\prime}}$ is adjacent to $x_{q}$. Therefore, the first outcome of the theorem holds with $e_{1}$ being $x_{q}-x_{r}$. Now we may assume that for every 
bad shortcut $Q$ across $S_{1}$ with $\left|N\left(\beta_{Q}\right) \cap V(C)\right|=1$ and $R$ across $T_{1}$ with $\left|N\left(\beta_{R}\right) \cap V(C)\right|=1$, if $\left\{x_{q}\right\}=N\left(\beta_{Q}\right) \cap V(C)$ and $\left\{x_{r}\right\}=N\left(\beta_{R}\right) \cap V(C)$, then $x_{q}=x_{r}$. By the first outcome of 3.3, for any bad shortcut $Q^{\prime}$ across $S_{1}$ with $\left|N\left(\beta_{Q^{\prime}}\right) \cap V(C)\right|>1, \beta_{Q^{\prime}}$ is adjacent to $x_{q}$, and for any bad shortcut $R^{\prime}$ across $T_{1}$ with $\left|N\left(\beta_{R^{\prime}}\right) \cap V(C)\right|>1, \beta_{R^{\prime}}$ is adjacent to $x_{r}$, which equals $x_{q}$. By (4) with $p=x_{q}$, the first outcome of the theorem holds.

Next assume that there exists a bad shortcut $Q$ across $S_{1}$ with $\mid N\left(\beta_{Q}\right) \cap$ $V(C) \mid=1$. Then we may assume because of the previous paragraph that for every bad shortcut $R$ across $T_{1},\left|N\left(\beta_{R}\right) \cap V(C)\right|>1$. The first outcome of 3.3 holds for shortcuts across $S_{1}$ and the second outcome of 3.3 holds for shortcuts across $T_{1}$. Let $\left\{x_{q}\right\}=N\left(\beta_{Q}\right) \cap V(C)$. Suppose there is a bad shortcut $Q^{\prime}$ across $S_{1}$ with $N\left(\beta_{Q^{\prime}}\right) \cap V(C)=\left\{x_{q^{\prime}}\right\}$ such that $x_{q^{\prime}} \neq x_{q}$. By the first outcome of $3.3, x_{q}$ is adjacent to $x_{q}^{\prime}$ and for every bad shortcut $Q^{\prime \prime}$ across $S_{1}$, if $\left|N\left(\beta_{Q^{\prime \prime}}\right) \cap V(C)\right|=1$ then $\beta_{Q^{\prime \prime}}$ is adjacent to either $x_{q}$ or to $x_{q^{\prime}}$, and if $\left|N\left(\beta_{Q^{\prime \prime}}\right) \cap V(C)\right|>1$ then $\beta_{Q^{\prime \prime}}$ is adjacent to both $x_{q}$ and $x_{q^{\prime}}$. By (3), for every bad shortcut $R^{\prime}$ across $T_{1}$, both $x_{q}$ and $x_{q^{\prime}}$ are adjacent to $\beta_{R^{\prime}}$. Then the first outcome of the theorem holds with $e_{1}$ being the edge $x_{q^{-}} x_{q^{\prime}}$. So for every bad shortcut $Q^{\prime}$ across $S_{1}$ with $\left|N\left(\beta_{Q^{\prime}}\right) \cap V(C)\right|=1$ we may assume that $\beta_{Q^{\prime}}$ is adjacent to $x_{q}$. By the first outcome of 3.3, for any bad shortcut $Q^{\prime}$ across $S_{1}$ with $\left|N\left(\beta_{Q^{\prime}}\right) \cap V(C)\right|>1, \beta_{Q^{\prime}}$ is adjacent to $x_{q}$. By (3), for any bad shortcut $R^{\prime}$ across $T_{1}, \beta_{R^{\prime}}$ is adjacent to $x_{q}$. Therefore, by (4) with $p=x_{q}$, the first outcome of the theorem holds.

If there exists a bad shortcut $R$ across $T_{1}$ with $\left|N\left(\beta_{R}\right) \cap V(C)\right|=1$, then by symmetry, the argument of the previous paragraph applies. So we may finally assume that for every shortcut $P$ across either $S_{1}$ or $T_{1}$, $\left|N\left(\beta_{P}\right) \cap V(C)\right|>1$. Then the second outcome of 3.3 holds for both $S_{1}$ and $T_{1}$. Let $Q$ be a bad shortcut across $S_{1}$ with $N(Q) \cap V(C) \subseteq P_{2}^{*}$. Let $c_{1}-p-c_{2}$ be a subpath of $P_{2}$ such that $N(Q) \cap V(C)=\left\{c_{1}, p, c_{2}\right\}$. By the second outcome of 3.3 , for any bad shortcut $Q^{\prime}$ across $S_{1}$ with $N\left(\beta_{Q^{\prime}}\right) \cap V(C) \subseteq P_{2}^{*}$, $\beta_{Q^{\prime}}$ is adjacent to $p$. Also, by (2), for any bad shortcut $R$ across $T_{1}$ with $N\left(\beta_{R}\right) \cap V(C) \subseteq P_{2}^{*}, \beta_{R}$ is adjacent to $p$. By symmetry between $P_{2}$ and $P_{3}$, there is also a vertex $s \in P_{3}^{*}$ such that for every bad shortcut $R$ across $S_{1}$ or $T_{1}$ with $N\left(\beta_{R}\right) \cap V(C) \subseteq P_{3}^{*}, \beta_{R}$ is adjacent to $s$. Therefore, by (4), the second case of the theorem holds. This proves 3.4.

3.5. There is an algorithm with the following specifications:

- Input: $A$ graph $G$.

- Output: A sequence of subsets $X_{1}, \ldots, X_{r}$ of $V(G)$ with $r \leq 2|V(G)|^{4}$, such that for every almost clean smallest prism $K$ in $G$ and $i \in$ 
$\{1,2,3\}$, one of $X_{1}, \ldots, X_{r}$ is disjoint from $V(K)$ and contains a vertex of every bad shortcut across the half-paths $S_{i}$ and $T_{i}$ of $K$.

- Running Time: $O\left(|V(G)|^{5}\right)$.

Proof. The algorithm is as follows. Enumerate all quadruples of distinct vertices $\left(u_{1}, u_{2}, u_{3}, u_{4}\right)$. For each, define the subset

$$
Y=\left(N\left(u_{2}\right) \cup N\left(u_{3}\right)\right) \backslash\left\{u_{1}, u_{2}, u_{3}, u_{4}\right\}
$$

and the subset

$$
Z=N\left(u_{1}, u_{2}\right) \cup N\left(u_{3}, u_{4}\right) .
$$

Let $Y_{1}, \ldots, Y_{s}$ and $Z_{1}, \ldots, Z_{s}$ be the subsets generated. Output $\emptyset, Y_{1}, \ldots, Y_{s}$, $Z_{1}, \ldots, Z_{s}$.

That concludes the description of the algorithm; we now prove that it works correctly. It takes time $O\left(|V(G)|^{5}\right)$ to find the sets $Y_{1}, \ldots, Y_{s}$ and $Z_{1}, \ldots, Z_{s}$ and the number of subsets in the output is $\leq 2|V(G)|^{4}$. It remains to check that the output sequence has the properties claimed. Let $K$ be an almost clean smallest prism in $G$ with half-paths $S_{1}, T_{1}, S_{2}, T_{2}, S_{3}$ and $T_{3}$. We may assume by symmetry that $i=1$. Apply 3.4 to $S_{1}$ and $T_{1}$.

If the first outcome of 3.4 holds, then there is an edge in $P_{2}^{*} \cup P_{3}^{*}$, say $u_{2}-u_{3}$, such that every bad shortcut across $S_{1}$ or $T_{1}$ contains an interior vertex adjacent to either $u_{2}$ or $u_{3}$. Choose $u_{1}$ to be the neighbor of $u_{2}$ in $V(K) \backslash\left\{u_{3}\right\}$ and $u_{4}$ to be the neighbor of $u_{3}$ in $V(K) \backslash\left\{u_{2}\right\}$. Then the subset $\left(N\left(u_{2}\right) \cup N\left(u_{3}\right)\right) \backslash\left\{u_{1}, u_{2}, u_{3}, u_{4}\right\}$ is disjoint from $V(K)$ and contains an interior vertex of every bad shortcut across $S_{1}$ or $T_{1}$.

If the second outcome of 3.4 holds, then there are two edges in $P_{2}^{*} \cup P_{3}^{*}$, say $u_{1}-u_{2}$ and $u_{3}-u_{4}$, such that the set $N\left(u_{1}, u_{2}\right) \cup N\left(u_{3}, u_{4}\right)$ contains an interior vertex of every bad shortcut across $S_{1}$ or $T_{1}$. Since $u_{1}$ and $u_{2}$ are adjacent, they have no common neighbors in $V(K)$, and since $u_{3}$ and $u_{4}$ are adjacent, they have no common neighbors in $V(K)$, so $N\left(u_{1}, u_{2}\right) \cup N\left(u_{3}, u_{4}\right)$ is disjoint from $V(K)$. This proves 3.5.

\section{Major vertices}

In this section, let $K$ be a smallest prism in a graph $G$, and assume that $K$ has a tidy frame. Let the vertices of $P_{1}$ be $f_{1}, f_{2}, \ldots, f_{n}$, numbered in order with $f_{1}=a_{1}$ and $f_{n}=b_{1}$. Let the vertices of $P_{2}$ be $h_{1}, h_{2}, \ldots, h_{m}$, numbered in order with $h_{1}=a_{2}$ and $h_{m}=b_{2}$, and let the vertices of $P_{3}$ be $g_{1}, g_{2}, \ldots, g_{p}$, numbered in order with $g_{1}=a_{3}$ and $g_{p}=b_{3}$. 
Recall that a $K$-major vertex is a vertex in $V(G) \backslash V(K)$ whose neighbors in $K$ are not contained in a 3-vertex path in $K$. Since $K$ has a tidy frame, every $K$-major vertex is anticomplete to $\left\{a_{1}, a_{2}, a_{3}, b_{1}, b_{2}, b_{3}\right\}$. For a $K$ major vertex $x$, if $x$ has neighbors in $P_{1}$, let $j_{x}$ be minimal such that $x$ is adjacent to $f_{j_{x}}$ and let $k_{x}$ be maximal such that $x$ is adjacent to $f_{k_{x}}$. Similarly, let $s_{x}$ be minimal and $t_{x}$ maximal such that $x$ is adjacent to $h_{s_{x}}$ and $h_{t_{x}}$, and let $c_{x}$ be minimal and $d_{x}$ maximal such that $x$ is adjacent to $g_{c_{x}}$ and $g_{d_{x}}$.

We start with a few easy but useful lemmas.

4.1. A K-major vertex has at least three neighbors in $K$.

Proof. Let $x$ be a major vertex with $N(x) \cap V(K)=\{u, v\}$. We may assume by symmetry that $\{u, v\} \subset V\left(P_{2}\right) \cup V\left(P_{3}\right)$. Since $x$ is major, $u$ and $v$ are not adjacent, so the subgraph induced by $G$ on $V\left(P_{2}\right) \cup V\left(P_{3}\right) \cup\{x\}$ is a theta smaller than $K$, a contradiction.

4.2. There is no $K$-major vertex with exactly two neighbors in $P_{2}$ and no neighbors in $P_{3}$.

Proof. Let $x$ be a $K$-major vertex with $N(x) \cap V\left(P_{2}\right)=\left\{h_{s_{x}}, h_{t_{x}}\right\}$ and $N(x) \cap$ $V\left(P_{3}\right)=\emptyset$. By 4.1, $x$ has at least one neighbor in $P_{1}$. If $s_{x}+1<t_{x}$, then the subgraph induced by $G$ on $V\left(P_{2}\right) \cup V\left(P_{3}\right) \cup\{x\}$ is a theta smaller than $K$, a contradiction. So we may assume that $s_{x}+1=t_{x}$. Now the triangles $\left\{h_{s_{x}}, h_{t_{x}}, x\right\}$ and $\left\{a_{1}, a_{2}, a_{3}\right\}$ and the paths $h_{s_{x}}-P_{2}-a_{2}, h_{t_{x}}-P_{2}-b_{2}-b_{3}-P_{3}-a_{3}$ and $x-f_{j_{x}}-P_{1}-a_{1}$ form a prism, $K^{\prime}$. Since $V\left(K^{\prime}\right) \subseteq V(K) \cup\{x\} \backslash\left\{f_{j_{x}+1}, b_{1}\right\}$, the prism $K^{\prime}$ is smaller than $K$ unless $f_{j_{x}+1}=b_{1}$. Also, the triangles $\left\{x, h_{s_{x}}, h_{t_{x}}\right\}$ and $\left\{b_{1}, b_{2}, b_{3}\right\}$ and the paths $h_{s_{x}}-P_{2}-a_{2}-a_{3}-P_{3}-b_{3}, h_{t_{x}}-P_{2}-b_{2}$ and $x-f_{k_{x}}-P_{1}-b_{1}$ form a prism, $K^{\prime \prime}$. Since $V\left(K^{\prime \prime}\right) \subseteq V(K) \cup\{x\} \backslash\left\{f_{k_{x}-1}, a_{1}\right\}$, the prism $K^{\prime \prime}$ is smaller than $K$ unless $f_{k_{x}-1}=a_{1}$. Therefore, since $K$ is a smallest prism, $f_{j_{x}+1}=b_{1}$ and $f_{k_{x}-1}=a_{1}$. It follows that $j_{x}=k_{x}$ and $f_{j_{x}}$ is adjacent to both $a_{1}$ and $b_{1}$, so $f_{j_{x}}=m_{1}$, contradicting the fact that $K$ has a tidy frame.

4.3. Let $x$ and $y$ be vertices each with at least two nonadjacent neighbors in a path $P=v_{1}-\cdots-v_{n}$. If there do not exist paths $A$ and $B$ between $x$ and $y$ with $A^{*} \subset V(P)$ and $B^{*} \subset V(P)$ such that $A^{*}$ and $B^{*}$ are anticomplete to each other, then for some $1 \leq i \leq n, N(x) \cap V(P) \subset\left\{v_{1}, \ldots, v_{i+1}\right\}$ and $N(y) \cap V(P) \subset\left\{v_{i}, \ldots, v_{n}\right\}$.

Proof. Let $v_{s}$ and $v_{t}$ be the neighbors of $x$ in $P$ with $s$ minimum and $t$ maximum. Let $v_{p}$ and $v_{r}$ be the neighbors of $y$ in $P$ with $p$ minimum and 
$r$ maximum. Since $x$ and $y$ each have two nonadjacent neighbors in $P$, $s+1<t$ and $p+1<r$. If $p<s$ and $r>t$ then there are paths $A$ and $B$ between $x$ and $y$ with $A^{*} \subseteq\left\{v_{p}, \ldots, v_{s}\right\}$ and $B^{*} \subseteq\left\{v_{t}, \ldots, v_{r}\right\}$ and $A^{*}$ and $B^{*}$ are anticomplete to each other, a contradiction. So either $p \geq s$ or $r \leq t$. By symmetry, we may assume that $p \geq s$. If $r \leq t$ then there are paths $A$ and $B$ between $x$ and $y$ with $A^{*} \subseteq\left\{v_{s}, \ldots, v_{p}\right\}$ and $B^{*} \subseteq\left\{v_{r}, \ldots, v_{t}\right\}$ and $A^{*}$ and $B^{*}$ are anticomplete to each other, a contradiction. Therefore, $r>t$. If $p<t-1$ then there are paths $A$ and $B$ between $x$ and $y$ with $A^{*} \subseteq\left\{v_{s}, \ldots, v_{p}\right\}$ and $B^{*} \subseteq\left\{v_{t}, \ldots, v_{r}\right\}$ and $A^{*}$ and $B^{*}$ are anticomplete to each other, a contradiction. So $p \geq t-1$, and the theorem holds with $i=t-1$. This proves 4.3 .

4.4. Every $K$-major vertex has neighbors in at least two of $P_{1}, P_{2}$ and $P_{3}$.

Proof. Let $v$ be a $K$-major vertex with $N(v) \cap V(K) \subseteq V\left(P_{1}\right)$. Since $v$ is major, $j_{v}+2<k_{v}$. The subgraph induced by $G$ on $V(K) \cup\{v\} \backslash$ $\left\{f_{j_{v}+1}, \ldots, f_{k_{v}-1}\right\}$ is a prism that is smaller than $K$. Therefore, $N(v) \cap$ $V(K) \nsubseteq V\left(P_{1}\right)$. By symmetry between $P_{1}, P_{2}$ and $P_{3}$, it follows that $N(v) \cap V(K) \nsubseteq V\left(P_{2}\right)$ and $N(v) \cap V(K) \nsubseteq V\left(P_{3}\right)$. This proves 4.4.

4.5. Let $x$ and $y$ be $K$-major vertices that are not adjacent to each other. Then for some $i, j$ with $1 \leq i<j \leq 3, N(x) \cap V(K) \subseteq V\left(P_{i}\right) \cup V\left(P_{j}\right)$ and $N(y) \cap V(K) \subseteq V\left(P_{i}\right) \cup V\left(P_{j}\right)$.

Proof. We first prove that each of $x$ and $y$ has neighbors in exactly two of $P_{1}, P_{2}$ and $P_{3}$.

(1) None of $x$ and $y$ has neighbors in all of $P_{1}, P_{2}, P_{3}$.

Assume that $x$ and $y$ both have neighbors in all three of $P_{1}, P_{2}$ and $P_{3}$. Then there are three paths between $x$ and $y$ each with interior in $P_{i}^{*}$ for $i \in$ $\{1,2,3\}$. These paths form a theta whose vertex set is contained in $(V(K) \backslash$ $\left.\left\{a_{1}, a_{2}, a_{3}, b_{1}, b_{2}, b_{3}\right\}\right) \cup\{x, y\}$ and is thus smaller than $K$, a contradiction. Therefore, at least one of $x$ and $y$ does not have neighbors in all three paths $P_{1}, P_{2}$ and $P_{3}$.

We may assume by symmetry that $N(y) \cap V\left(P_{1}\right)=\emptyset$. Also, we may assume that $x$ has neighbors in all of $P_{1}, P_{2}$ and $P_{3}$, because otherwise (1) holds. By 4.4, $y$ has neighbors in both $P_{2}$ and $P_{3}$. By 4.1, it has at least two neighbors in one of these paths, say $P_{2}$. Then by 4.2 , it has two nonadjacent neighbors in $P_{2}$.

Since $x$ and $y$ both have neighbors in $P_{3}$, there is a path $R$ between them with interior in $P_{3}^{*}$. Let $P$ be the path $h_{s_{y}}-P_{2}-a_{2}-a_{1}-P_{1}-b_{1}-b_{2}-P_{2}-h_{t_{y}}$. Assume 
that $x$ has two nonadjacent neighbors in $P$. Then by 4.3 , there are two paths $R^{\prime}$ and $R^{\prime \prime}$ between $x$ and $y$ whose interiors are anticomplete to each other and contained in $V(P)$. Since $V(P)$ is anticomplete to $P_{3}^{*}$, the paths $x-R-y, x-R^{\prime}-y$ and $x-R^{\prime \prime}-y$ form a theta, $K^{\prime}$. Since $V\left(K^{\prime}\right) \subseteq V(K) \cup\{x, y\} \backslash$ $\left\{a_{3}, b_{3}, h_{s_{y}+1}\right\}, K^{\prime}$ is smaller than $K$, a contradiction. Therefore, $x$ does not have two nonadjacent neighbors in $P$. Since $P_{1}$ is a subpath of $P$, this implies that $x$ does not have two nonadjacent neighbors in $P_{1}$, so $j_{x} \geq k_{x}-1$. Also, since $N(x) \cap P_{1}^{*} \neq \emptyset$, it follows that $N(x) \cap\left(V(P) \backslash V\left(P_{1}\right)\right)=\emptyset$. Thus $s_{x}>s_{y}$ and $t_{x}<t_{y}$.

If $s_{x}<t_{y}-1$ then the paths $x-R-y, x-f_{k_{x}}-P_{1}-b_{1}-b_{2}-P_{2}-h_{t_{y}}-y$, and a path between $x$ and $y$ with interior in $\left\{h_{s_{y}}, \ldots, h_{s_{x}}\right\}$ form a theta, $K^{\prime}$. Since $V\left(K^{\prime}\right) \subseteq V(K) \cup\{x, y\} \backslash\left\{h_{t_{y}-1}, a_{3}, b_{3}\right\}, K^{\prime}$ is smaller than $K$, a contradiction. Therefore, $s_{x} \geq t_{y}-1$. Since $s_{x} \leq t_{x}$ and $t_{x}<t_{y}$, this proves that $s_{x}=t_{y}-1=t_{x}$. If $t_{x}>s_{y}+1$, then the paths $x-R-y, x-f_{j_{x}}-P_{1}-a_{1}-a_{2}-P_{2}-h_{s_{y}}-y$ and a path between $x$ and $y$ with interior in $\left\{h_{t_{x}}, h_{t_{y}}\right\}$ form a theta, $K^{\prime}$. Since $V\left(K^{\prime}\right) \subseteq V(K) \cup\{x, y\} \backslash\left\{h_{s_{y}+1}, a_{3}, b_{3}\right\}, K^{\prime}$ is smaller than $K$, a contradiction. Therefore, $t_{x} \leq s_{y}+1$. Since $t_{x}=s_{x}>s_{y}$, this proves that $s_{x}=t_{x}=s_{y}+1$. Therefore, $s_{y}+2=t_{y}$ and $N(x) \cap V\left(P_{2}\right)=\left\{h_{s_{y}+1}\right\}$.

If $y$ is not adjacent to $h_{s_{y}+1}$ then $\left|N(y) \cap V\left(P_{2}\right)\right|=2$ and $N(y) \cap V\left(P_{1}\right)=$ $\emptyset$, contradicting 4.2. Therefore, $y$ is adjacent to $h_{s_{y}+1}$. If $j_{x}=k_{x}$ then the subgraph induced by $G$ on $V\left(P_{1}\right) \cup V\left(P_{2}\right) \cup\{x\}$ is a theta smaller than $K$, a contradiction. Therefore, $j_{x}<k_{x}$, and since $j_{x} \geq k_{x}-1$, it follows that $j_{x}=$ $k_{x}-1$. If $c_{y}<c_{x}$ then the triangles $\left\{h_{s_{y}}, h_{s_{y}+1}, y\right\}$ and $\left\{a_{1}, a_{2}, a_{3}\right\}$ and the paths $h_{s_{y}}-P_{2^{-}} a_{2}, h_{s_{y}+1^{-}}-f_{j_{x}}-P_{1^{-}} a_{1}$ and $y-g_{c_{y}}-P_{3^{-}} a_{3}$ form a prism, $K^{\prime}$. Since $V\left(K^{\prime}\right) \subseteq V(K) \cup\{x, y\} \backslash\left\{b_{1}, b_{2}, b_{3}\right\}, K^{\prime}$ is smaller than $K$, a contradiction. Therefore $c_{y} \geq c_{x}$. If $c_{x}<c_{y}$ then the triangles $\left\{x, f_{j_{x}}, f_{k_{x}}\right\}$ and $\left\{a_{1}, a_{2}, a_{3}\right\}$ and the paths $x-g_{c_{x}}-P_{3}-a_{3}, f_{j_{x}}-P_{1}-a_{1}$ and $f_{k_{x}}-P_{1}-b_{1}-b_{2}-P_{2}-h_{t_{y}}-y-h_{s_{y}}-P_{2^{-}} a_{2}$ form a prism, $K^{\prime}$. Since $V\left(K^{\prime}\right) \subseteq V(K) \cup\{x, y\} \backslash\left\{h_{s_{y}+1}, b_{3}, g_{c_{y}}\right\}, K^{\prime}$ is smaller than $K$, a contradiction. Therefore, $c_{x}=c_{y}$. By the symmetry of the prism $K$, it follows by the same argument that $d_{x}=d_{y}$.

If $c_{x}=d_{x}$ then the induced subgraph with vertex set $V\left(P_{2}\right) \cup V\left(P_{3}\right) \cup\{x\}$ is a theta smaller than $K$, a contradiction. So $c_{x}<d_{x}$, which means that $c_{y}<d_{y}$. If $c_{y}<d_{y}-1$ then the paths $x-g_{c_{y}}-y, x-g_{d_{y}}-y$ and $x-h_{s_{y}+1^{-}}-y$ form a theta smaller than $K$, a contradiction. Therefore, $c_{y}=d_{y}-1$. But then $\left|N(y) \cap V\left(P_{3}\right)\right|=2$ and $N(y) \cap V\left(P_{1}\right)=\emptyset$, which contradicts 4.2. This proves (1).

By 4.4 and (1), each of $x$ and $y$ has neighbors in precisely two of the paths $P_{1}, P_{2}$ and $P_{3}$. Assume that $N(x) \cap V(K)$ and $N(y) \cap V(K)$ are not 
both contained in the union of the same two of $V\left(P_{1}\right), V\left(P_{2}\right)$ and $V\left(P_{3}\right)$. By symmetry, we may assume that $N(x) \cap V\left(P_{3}\right)=\emptyset$ and that $N(y) \cap V\left(P_{1}\right)=\emptyset$.

Assume that $j_{x}<k_{x}-1$ and $c_{y}<d_{y}-1$; this means that $x$ has two nonadjacent neighbors in $P_{1}$ and $y$ has two nonadjacent neighbors in $P_{3}$. Since both $x$ and $y$ have neighbors in $P_{2}$, there exists a path $R$ between $x$ and $y$ with interior in $P_{2}^{*}$. Then the three paths $x-f_{j_{x}}-P_{1}-a_{1}-a_{3}-P_{3}-g_{c_{y}}-y$, $x-f_{k_{x}}-P_{1}-b_{1}-b_{3}-P_{3}-g_{d_{y}}-y$ and $x-R-y$ form a theta, $K^{\prime}$. Since $V\left(K^{\prime}\right) \subseteq V(K) \cup$ $\{x, y\} \backslash\left\{a_{2}, b_{2}, f_{j_{x}+1}\right\}, K^{\prime}$ is smaller than $K$, a contradiction. This implies that either $x$ does not have two nonadjacent neighbors in $P_{1}$ or $y$ does not have two nonadjacent neighbors in $P_{3}$. By symmetry we may assume that $x$ does not have two nonadjacent neighbors in $P_{1}$, so $j_{x} \geq k_{x}-1$. By 4.2, $N(x) \cap V\left(P_{1}\right)$ does not consist of two adjacent vertices. Therefore, $\left|N(x) \cap V\left(P_{1}\right)\right|=1$, and $j_{x}=k_{x}$.

Since $x$ is $K$-major, by $4.1,\left|N(x) \cap V\left(P_{2}\right)\right| \geq 2$ and by $4.2, N(x) \cap V\left(P_{2}\right)$ does not consist of two adjacent vertices. Therefore, $x$ has two nonadjacent neighbors in $P_{2}$, and $s_{x}<t_{x}-1$.

First assume that $\left|N(y) \cap V\left(P_{2}\right)\right|=1$. Then $s_{y}=t_{y}$, and by 4.2 and 4.1, $y$ has two nonadjacent neighbors in $P_{3}$, so $c_{y}<d_{y}-1$. If $s_{y}<t_{x}-1$ then the paths $y-g_{d_{y}}-P_{3}-b_{3}-b_{2}-P_{2}-h_{t_{x}}-x, y-g_{c_{y}}-P_{3}-a_{3}-a_{1}-P_{1}-f_{j_{x}}-x$, and a path between $x$ and $y$ with interior in $V\left(h_{s_{y}}-P_{2}-h_{s_{x}}\right)$ form a theta, $K^{\prime}$. Since $V\left(K^{\prime}\right) \subseteq V(K) \cup\{x, y\} \backslash\left\{h_{t_{x}-1}, a_{2}, b_{1}\right\}, K^{\prime}$ is smaller than $K$, a contradiction. Therefore, $s_{y} \geq t_{x}-1$. From the symmetry, $s_{y} \leq s_{x}+1$. So we have that $t_{x}-1 \leq s_{y} \leq s_{x}+1$ and $s_{x}<t_{x}-1$, from which it follows that $s_{x}+1=s_{y}=t_{x}-1$. If $x$ is not adjacent to $h_{s_{x}+1}$ then $\left|N(x) \cap V\left(P_{2}\right)\right|=2$ and $N(x) \cap V\left(P_{3}\right)=\emptyset$, contradicting 4.2. Therefore, $x$ is adjacent to $h_{s_{x}+1}$. The triangles $\left\{h_{s_{x}}, h_{s_{x}+1}, x\right\}$ and $\left\{a_{1}, a_{2}, a_{3}\right\}$ and the paths $h_{s_{x}}-P_{2}-a_{2}, h_{s_{x}+1}-y-g_{c_{y}}-P_{3}-a_{3}$ and $x-f_{j_{x}}-P_{1}-a_{1}$ form a prism, $K^{\prime}$. Since $V\left(K^{\prime}\right) \subseteq V(K) \cup\{x, y\} \backslash\left\{b_{1}, b_{2}, b_{3}\right\}, K^{\prime}$ is smaller than $K$, a contradiction. This proves that $\left|N(y) \cap V\left(P_{2}\right)\right|>1$.

By 4.2, $y$ has more than two neighbors in $P_{2}$, and $s_{y}<t_{y}-1$. Let $Q$ be the path $x-f_{j_{x}}-P_{1}-a_{1}-a_{3}-P_{3}-g_{c_{y}}-y$. If there are two paths $A$ and $B$ between $x$ and $y$ whose interiors are anticomplete to each other and contained in $P_{2}^{*}$, then the paths $Q, A$ and $B$ form a theta, $K^{\prime}$. Since $V\left(K^{\prime}\right) \subseteq V(K) \cup\{x, y\} \backslash$ $\left\{b_{1}, b_{2}, b_{3}\right\}, K^{\prime}$ is smaller than $K$, a contradiction. Therefore, there are no two paths between $x$ and $y$ whose interiors are anticomplete to each other and contained in $P_{2}^{*}$. Then by 4.3 , either $N(y) \cap V\left(P_{2}\right) \subseteq\left\{h_{1}, \ldots, h_{s_{x}+1}\right\}$ or $N(y) \cap V\left(P_{2}\right) \subseteq\left\{h_{t_{x}-1}, \ldots, h_{m}\right\}$. By symmetry, we may assume that $N(y) \cap V\left(P_{2}\right) \subseteq\left\{h_{1}, \ldots, h_{s_{x}+1}\right\}$, which means that $t_{y} \leq s_{x}+1$.

If $t_{y} \leq s_{x}$, then the paths $y-h_{s_{y}}-P_{2}-a_{2}-a_{1}-P_{1}-f_{j_{x}}-x, y-g_{d_{y}}-P_{3}-b_{3}-b_{2}-P_{2}-h_{t_{x}}-x$, and a path between $x$ and $y$ with interior in $V\left(h_{t_{y}}-P_{2}-h_{s_{x}}\right)$ form a theta, 
$K^{\prime}$. Since $V\left(K^{\prime}\right) \subseteq V(K) \cup\{x, y\} \backslash\left\{a_{3}, b_{1}, h_{s_{x}+1}\right\}, K^{\prime}$ is smaller than $K$, a contradiction. Therefore, $t_{y}>s_{x}$, and since $t_{y} \leq s_{x}+1$, it follows that $t_{y}=s_{x}+1$ and $y$ is adjacent to $h_{s_{x}+1}$.

Assume that $s_{y}<s_{x}-1$ and $t_{x}>t_{y}+1$. Then the paths $x-f_{j_{x}}-P_{1}-a_{1}-a_{2}-P_{2}-h_{s_{y}}-y$, $x-h_{t_{x}}-P_{2}-b_{2}-b_{3}-P_{3}-g_{d_{y}}-y$ and a path between $x$ and $y$ with interior in $\left\{h_{s_{x}}, h_{s_{x}+1}\right\}$ form a theta, $K^{\prime}$. Since $V\left(K^{\prime}\right) \subseteq V(K) \cup\{x, y\} \backslash\left\{a_{3}, h_{s_{x}-1}, h_{t_{x}-1}\right\}, K^{\prime}$ is smaller than $K$, a contradiction. Therefore, either $s_{y} \geq s_{x}-1$ or $t_{x} \leq t_{y}+1$.

First assume that $s_{y} \geq s_{x}-1$. Then since $s_{x}=t_{y}-1$ and $s_{y}<t_{y}-1$, this implies that $s_{y}=s_{x}-1$. If $y$ is adjacent to $h_{s_{x}}$ then the triangles $\left\{h_{s_{x}-1}, h_{s_{x}}, y\right\}$ and $\left\{a_{1}, a_{2}, a_{3}\right\}$ and the paths $h_{s_{x}-1}-P_{2}-a_{2}, h_{s_{x}}-x-f_{j_{x}}-P_{1}-a_{1}$ and $y-g_{c_{y}}-P_{3}-a_{3}$ form a prism, $K^{\prime}$. Since $V\left(K^{\prime}\right) \subseteq V(K) \cup\{x, y\} \backslash\left\{b_{1}, b_{2}, b_{3}\right\}$, $K^{\prime}$ is smaller than $K$, a contradiction. Therefore, $y$ is not adjacent to $h_{s_{x}}$. Then since $t_{y}=s_{y}+2$, this means that $\left|N(y) \cap V\left(P_{2}\right)\right|=2$, which contradicts 4.2. This proves that $s_{y}<s_{x}-1$, so we may now assume that $t_{x} \leq t_{y}+1$. Since $t_{y}=s_{x}+1$ and $t_{x}>s_{x}+1$, this implies that $t_{x}=s_{x}+2$. If $x$ is adjacent to $h_{s_{x}+1}$ then the triangles $\left\{h_{s_{x}+1}, h_{t_{x}}, x\right\}$ and $\left\{b_{1}, b_{2}, b_{3}\right\}$ and the paths $h_{s_{x}+1}-y-g_{d_{y}}-P_{3}-b_{3}, h_{t_{x}}-P_{2}-b_{2}$, and $x-f_{k_{x}}-P_{1}-b_{1}$ form a prism, $K^{\prime}$. Since $V\left(K^{\prime}\right) \subseteq V(K) \cup\{x, y\} \backslash\left\{a_{1}, a_{2}, a_{3}\right\}, K^{\prime}$ is smaller than $K$, a contradiction. Therefore, $x$ is not adjacent to $h_{s_{x}+1}$. Then since $t_{x}=s_{x}+2$, this means that $\left|N(x) \cap V\left(P_{2}\right)\right|=2$, which contradicts 4.2. This proves 4.5.

4.6. Two $K$-major vertices that are not adjacent have a common neighbor in $K$.

Proof. Let $x$ and $y$ be $K$-major vertices that are not adjacent to each other. Assume that $N(x) \cap V(K) \cap N(y)=\emptyset$. From 4.5 we may assume that $(N(x) \cup N(y)) \cap V(K) \subset P_{1}^{*} \cup P_{2}^{*}$. By 4.4, each of $P_{1}^{*}$ and $P_{2}^{*}$ contains a neighbor of $x$ and a neighbor of $y$. Let $C$ be the cycle induced by $G$ on $V\left(P_{1}\right) \cup V\left(P_{2}\right)$. A gap is a minimal path in $C$ containing a neighbor of $x$ and a neighbor of $y$. Every edge of $C$ is in at most one gap and any vertex common to two distinct gaps is an end of both. Since $x$ and $y$ have no common neighbor in $C$, there is an even number of gaps. For a gap $P$, let $P^{+}$be the path between $x$ and $y$ whose interior is $V(P)$. Let $R_{1}, \ldots, R_{k}$ be the gaps of $C$ numbered in their order on $C$, such that the vertex set of the path of $C$ sharing one end with $R_{i}$ and the other end with $R_{i+1}$, and with interior disjoint from all the gaps, is anticomplete to $y$ if $i$ is odd and anticomplete to $x$ if $i$ is even. Call a gap a small gap if it consists of one edge and shares both of its ends with other gaps.

(1) There are at most six gaps. 
Suppose there are at least eight gaps. Assume that for some $i, R_{i}$ is not a small gap. Then the paths $R_{i+1}^{+}, R_{i+4}^{+}$and $R_{i+7}^{+}$form a theta, $K^{\prime}$. It is smaller than $K$ because each of $R_{i}, R_{i+2} \cup R_{i+3}$ and $R_{i+5} \cup R_{i+6}$ contains at least one vertex not in $V\left(K^{\prime}\right)$. Therefore, every gap is small. Then every edge of $C$ is in a gap and every gap consists of one edge, so $V(C) \subseteq N(x) \cup N(y)$. But this contradicts the fact that $x$ and $y$ have no neighbors in $\left\{a_{1}, a_{2}, b_{1}, b_{2}\right\} \subseteq C$. This proves (1).

\section{(2) There are at most four gaps.}

Suppose there are six gaps. Assume that no gap is small. The paths $R_{1}^{+}, R_{3}^{+}$and $R_{5}^{+}$form a theta. It is smaller than $K$ because $R_{2}, R_{4}$ and $R_{6}$ each contain at least one vertex not in the theta since they are not small gaps. So there is a small gap; by symmetry let it be $R_{1}$. Assume next that none of $R_{2}, R_{4}$ or $R_{6}$ is a small gap. Then the paths $R_{1}^{+}, R_{3}^{+}$and $R_{5}^{+}$form a theta smaller than $K$. Therefore one of $R_{2}, R_{4}$ or $R_{6}$ is small.

First assume that $R_{2}$ is a small gap. Then $R_{1} \cup R_{2}$ is a three-vertex path whose vertices are contained in $N(x) \cup N(y)$, and so it is a subpath of either $P_{1}$ or $P_{2}$ in $K$, because $x$ and $y$ have no neighbors in $\left\{a_{1}, a_{2}, b_{1}, b_{2}\right\}$. By symmetry assume it is a subpath of $P_{1}$. Both $x$ and $y$ have a neighbor in $P_{2}$, so there is a path $Q$ between them with interior in $P_{2}$. Let $u$ be the vertex that is an end of both $R_{1}$ and $R_{2}$. The three paths $u-R_{1}^{+}-y, u-R_{2}^{+}-y$ and $u-x-Q-y$ form a theta, $K^{\prime}$. Since $V\left(K^{\prime}\right)$ is disjoint from $\left\{a_{1}, a_{2}, a_{3}, b_{1}, b_{2}, b_{3}\right\}$, it follows that the theta $K^{\prime}$ is smaller than $K$, a contradiction. This proves that $R_{2}$ is not a small gap. From the symmetry, we deduce that $R_{6}$ is not a small gap either.

Therefore $R_{4}$ is a small gap. From the argument in the previous paragraph with $R_{4}$ playing the role of $R_{1}$, we deduce that neither of $R_{3}, R_{5}$ is a small gap. Since $R_{1}$ is a small gap and $R_{5}$ is not, we deduce that $V\left(R_{1}\right) \cap V\left(R_{6}\right) \neq \emptyset$, and that $V\left(R_{6}\right)$ is anticomplete to $V\left(R_{4}\right)$. Let $V\left(R_{1}\right) \cap$ $V\left(R_{6}\right)=\{v\}$. The paths $x-R_{4}^{+}-y-v, R_{6}^{+} \backslash y$, and $R_{1}^{+} \backslash y$ form a theta smaller than $K$, a contradiction.

This proves (2).

\section{(3) There are at most two gaps.}

Assume there are four gaps. Let $Q_{1}, \ldots, Q_{4}$ be subpaths of $C$ such that none of their interiors meet any gap, and such that for $1 \leq i \leq 3$, one end of $Q_{i}$ is an end of $R_{i}$ and the other is an end of $R_{i+1}$, while the ends of $Q_{4}$ are ends of $R_{1}$ and $R_{4}$. The neighbors of $x$ in $C$ are contained in $V\left(Q_{1}\right) \cup V\left(Q_{3}\right)$ and the neighbors of $y$ in $C$ are contained in $V\left(Q_{2}\right) \cup V\left(Q_{4}\right)$. 
Assume that $Q_{1}$ and $Q_{3}$ both have length at most one. Then since $x$ has neighbors in both $P_{1}$ and $P_{2}$ but no neighbors in $\left\{a_{1}, a_{2}, b_{1}, b_{2}\right\}$, one of $Q_{1}$ and $Q_{3}$ is a subpath of $P_{1}$ and the other is a subpath of $P_{2}$. But then $x$ has at most two neighbors in each of $P_{1}$ and $P_{2}$, contradicting 4.1 or 4.2. This shows that at least one of $Q_{1}$ and $Q_{3}$ has length at least two. By symmetry, we may assume that $Q_{1}$ does. Therefore $V\left(R_{1}\right)$ is anticomplete to $V\left(R_{2}\right)$.

By the same argument applied to $Q_{2}$ and $Q_{4}$, we deduce that at least one of $Q_{2}$ and $Q_{4}$ has length at least two. From the symmetry, we may assume that $Q_{2}$ has length at least two, and therefore $V\left(R_{2}\right)$ is anticomplete to $V\left(R_{3}\right)$. Since the paths $R_{1}^{+}, R_{2}^{+}$and $R_{3}^{+}$do not form a theta smaller than $K$, it follows that $\left|V\left(Q_{3}\right)\right|=\left|V\left(Q_{4}\right)\right|=1$ and $R_{4}$ is a small gap. Let $V\left(Q_{3}\right)=\left\{q_{3}\right\}$ and $V\left(Q_{4}\right)=\left\{q_{4}\right\}$. Since $V\left(R_{2}\right)$ is anticomplete to both $V\left(R_{1}\right)$ and $V\left(R_{3}\right)$, the paths $x-q_{3}-q_{4}, R_{1}^{+} \backslash y$, and $x-R_{2}^{+}-y-q_{4}$ form a theta smaller that $K$, a contradiction. This proves (3).

Since $x$ and $y$ each have a neighbor in $C$, there are at least two gaps; so from (3) there are two gaps. Since $x$ and $y$ each have neighbors in both $P_{1}$ and $P_{2}$, there is a gap contained in $P_{1}$ and a gap contained in $P_{2}$. The subgraph $C \backslash\left(R_{1} \cup R_{2}\right)$ is the disjoint union of two paths, $Q_{1}$ and $Q_{2}$, such that $Q_{1}$ contains no neighbors of $x$ and $Q_{2}$ contains no neighbors of $y$. By 4.1 and 4.2, each of $x$ and $y$ has two nonadjacent neighbors in one of $P_{1}$ and $P_{2}$, so each of $x$ and $y$ has a neighbor in $C$ that is anticomplete to $V\left(R_{1}\right) \cup V\left(R_{2}\right)$. Therefore, there is a path $S$ between $x$ and $y$ whose interior is anticomplete to $V\left(R_{1}\right) \cup V\left(R_{2}\right)$ and is contained in $V(K) \backslash\left(V\left(R_{1}\right) \cup V\left(R_{2}\right)\right)$. The paths $S, R_{1}^{+}$and $R_{2}^{+}$form a theta smaller than $K$, a contradiction. This proves 4.6.

A broom is a vertex $v \in V(G)$ such that for some $\left\{i_{1}, i_{2}, i_{3}\right\}=\{1,2,3\}$,

- $N(v) \cap V\left(P_{i_{1}}\right)=\emptyset$,

- $\left|N(v) \cap V\left(P_{i_{2}}\right)\right|=1$, and

- $N(v) \cap V\left(P_{i_{3}}\right)$ is the vertex set of a three-vertex path.

4.7. If $x$ and $y$ are two $K$-major vertices that are not adjacent to each other, then one of them is a broom, and for some $i, j$ such that $1 \leq i<j \leq 3$, $N(x) \cap V\left(P_{i}\right) \cap N(y) \neq \emptyset$ and $N(x) \cap V\left(P_{j}\right) \cap N(y) \neq \emptyset$.

Proof. By 4.5, we may assume that $N(x) \cap V(K) \subseteq P_{1}^{*} \cup P_{2}^{*}$ and $N(y) \cap$ $V(K) \subseteq P_{1}^{*} \cup P_{2}^{*}$. By 4.4, $x$ and $y$ each have a neighbor in both $P_{1}^{*}$ and in $P_{2}^{*}$. Also, by 4.6, $x$ and $y$ have a common neighbor in $K$, and by the 
symmetry between $P_{1}$ and $P_{2}$ we may assume that $f_{r} \in P_{1}^{*}$ is a neighbor of both $x$ and $y$.

Let $A$ be the path induced by $G$ on $V\left(P_{1}\right) \cup V\left(P_{2}\right) \backslash\left\{f_{r-1}, f_{r}, f_{r+1}\right\}$.

(1) $x$ and $y$ do not both have a neighbor in $V(A) \cap V\left(P_{1}\right)$.

Suppose both $x$ and $y$ have a neighbor in $V(A) \cap V\left(P_{1}\right)$. Then there is a path, $S$, between $x$ and $y$ with interior in $\left(V(A) \cap V\left(P_{1}\right)\right) \cup V\left(P_{3}\right)$. Since $x$ and $y$ each have a neighbor in $P_{2}^{*}$ there is a path, $R$, between them with interior in $P_{2}^{*}$. The paths $S, x-f_{r^{-}} y$ and $R$ form a theta, $K^{\prime}$. Since $V\left(K^{\prime}\right) \subseteq V(K) \cup\{x, y\} \backslash\left\{f_{r-1}, f_{r+1}, a_{2}, b_{2}\right\}, K^{\prime}$ is smaller than $K$, a contradiction. This proves (1).

(2) If exactly one of $x$ and $y$ has a neighbor in $V(A) \cap V\left(P_{1}\right)$, then the theorem holds.

Assume that exactly one of $x$ and $y$ has neighbors in $V(A) \cap V\left(P_{1}\right)$. By symmetry between $x$ and $y$ we may assume that $N(x) \cap V(A) \cap V\left(P_{1}\right) \neq \emptyset$ and $N(y) \cap V(A) \cap V\left(P_{1}\right)=\emptyset$. By 4.1 and $4.2, y$ has two nonadjacent neighbors in at least one of $P_{1}$ and $P_{2}$. Since $y$ has no neighbors in $V(A) \cap V\left(P_{1}\right)$, this means that it either has two nonadjacent neighbors in $P_{2}$, so $t_{y}>s_{y}+1$, or it is adjacent to both $f_{r-1}$ and $f_{r+1}$.

First assume that $t_{y}>s_{y}+1$. Since $N(x) \cap V(A) \cap V\left(P_{1}\right) \neq \emptyset$ and $N(x) \cap$ $V\left(P_{2}\right) \neq \emptyset$, each of $x$ and $y$ has two nonadjacent neighbors in $A$. If there are two paths $S$ and $R$ between $x$ and $y$ whose interiors are anticomplete to each other and are contained in $V(A)$, then the paths $x-f_{r}-y, S$ and $R$ form a theta smaller than $K$, a contradiction. Therefore, by 4.3 we may assume that $N(x) \cap V(A) \subseteq V\left(a_{1}-P_{1}-f_{r-2}\right) \cup V\left(a_{2}-P_{2}-h_{s_{y}+1}\right)$, which means that $t_{x} \leq s_{y}+1$. Since $N(x) \cap V(A) \cap V\left(P_{1}\right) \neq \emptyset, j_{x}<r-1$. If $s_{x}<t_{y}-1$, then the paths $x-f_{r}-y, x-f_{j_{x}}-P_{1}-a_{1}-a_{3}-P_{3}-b_{3}-b_{2}-P_{2}-h_{t_{y}}-y$ and a path between $x$ and $y$ with interior in $V\left(h_{s_{x}}-P_{2}-h_{s_{y}}\right)$ form a theta, $K^{\prime}$. Since $V\left(K^{\prime}\right) \subseteq V(K) \cup\{x, y\} \backslash\left\{a_{2}, b_{1}, f_{r-1}\right\}, K^{\prime}$ is smaller than $K$, a contradiction. Therefore, $s_{x} \geq t_{y}-1$, and since $t_{x} \leq s_{y}+1$ and $t_{y}>s_{y}+1$, it follows that $s_{y}+1=t_{y}-1$ and $N(x) \cap V\left(P_{2}\right)=\left\{h_{s_{y}+1}\right\}$. By 4.2, $\left|N(y) \cap V\left(P_{2}\right)\right| \neq 2$, so $y$ is adjacent to $h_{s_{y}+1}$. Also by $4.2,\left|N(y) \cap V\left(P_{1}\right)\right| \neq 2$, so $y$ is either complete or anticomplete to $\left\{f_{r-1}, f_{r+1}\right\}$.

Suppose that $y$ is $\left\{f_{r-1}, f_{r+1}\right\}$-complete. If $x$ is not adjacent to $f_{r+1}$ then the triangles $\left\{f_{r}, f_{r+1}, y\right\}$ and $\left\{b_{1}, b_{2}, b_{3}\right\}$ and the paths $f_{r}-x-f_{j_{x}}-P_{1}-a_{1}-a_{3}-P_{3}-b_{3}$, $f_{r+1}-P_{1}-b_{1}$ and $y-h_{t_{y}}-P_{2}-b_{2}$ form a prism, $K^{\prime}$. Since $V\left(K^{\prime}\right) \subseteq V(K) \cup\{x, y\} \backslash$ $\left\{h_{s_{y}}, h_{s_{y}+1}, f_{r-1}\right\}, K^{\prime}$ is smaller than $K$, a contradiction. So $x$ is adjacent to $f_{r+1}$, and the paths $x-f_{r+1^{-}} y, x-h_{s_{y}+1^{-}} y$ and a path between $x$ and $y$ with 
interior in $V\left(f_{j_{x}}-P_{1}-f_{r-1}\right)$ form a theta smaller than $K$, a contradiction. Therefore, $y$ is anticomplete to $\left\{f_{r-1}, f_{r+1}\right\}$, and so $y$ is a broom and the theorem holds.

Since we have shown that the theorem holds when $t_{y}>s_{y}+1$, we may now assume that $t_{y} \leq s_{y}+1$ and that $y$ is $\left\{f_{r-1}, f_{r+1}\right\}$-complete. By 4.2, $\left|N(y) \cap V\left(P_{2}\right)\right| \neq 2$, so $t_{y}=s_{y}$. Since $N(x) \cap V(A) \cap V\left(P_{1}\right) \neq \emptyset$, either $j_{x}<r-1$ or $k_{x}>r+1$. By symmetry we may assume that $j_{x}<r-1$.

Suppose that $\left|N(x) \cap V\left(P_{2}\right)\right|=1$. If $x$ is adjacent to $f_{r+1}$ then the path $x-f_{r+1}-y$, a path between $x$ and $y$ with interior in $V\left(f_{j_{x}}-P_{1}-f_{r-1}\right)$ and a path between $x$ and $y$ with interior in $P_{2}^{*}$ form a theta smaller than $K$, a contradiction. Therefore, $x$ is not adjacent to $f_{r+1}$. If $s_{x}<s_{y}$ then the triangles $\left\{f_{r}, f_{r+1}, y\right\}$ and $\left\{b_{1}, b_{2}, b_{3}\right\}$ and the paths $f_{r}-x-f_{j_{x}}-P_{1}-a_{1}-a_{3}-P_{3}-b_{3}$, $f_{r+1}-P_{1}-b_{1}$ and $y-h_{s_{y}}-P_{2}-b_{2}$ form a prism, $K^{\prime}$. Since $V\left(K^{\prime}\right) \subseteq V(K) \cup$ $\{x, y\} \backslash\left\{f_{r-1}, a_{2}, h_{s_{x}}\right\}, K^{\prime}$ is smaller than $K$, a contradiction. If $s_{x}>s_{y}$, then the triangles $\left\{f_{r}, f_{r+1}, y\right\}$ and $\left\{a_{1}, a_{2}, a_{3}\right\}$ and the paths $f_{r-x-} f_{j_{x}}-P_{1}-a_{1}$, $f_{r+1}-P_{1}-b_{1}-b_{3}-P_{3}-a_{3}$ and $y-h_{s_{y}}-P_{2^{-}} a_{2}$ form a prism, $K^{\prime}$. Since $V\left(K^{\prime}\right) \subseteq$ $V(K) \cup\{x, y\} \backslash\left\{f_{r-1}, b_{2}, h_{s_{x}}\right\}, K^{\prime}$ is smaller than $K$, a contradiction. This proves that $s_{x}=s_{y}$, and $y$ is a broom, so the theorem holds. So we may assume that $\left|N(x) \cap V\left(P_{2}\right)\right|>1$, and it follows from 4.2 that $s_{x}<t_{x}-1$.

Let $Q$ be the path between $x$ and $y$ with interior in $V\left(f_{j_{x}}-P_{1}-f_{r-1}\right)$. If $t_{x} \leq s_{y}$ then the paths $Q, x-h_{t_{x}}-P_{2}-h_{s_{y}}-y$ and $x-h_{s_{x}}-P_{2}-a_{2}-a_{3}-P_{3}-b_{3}-b_{1}-P_{1}-f_{r+1}-y$ form a theta, $K^{\prime}$. Since $V\left(K^{\prime}\right) \subseteq V(K) \cup\{x, y\} \backslash\left\{f_{r}, a_{1}, b_{2}\right\}, K^{\prime}$ is smaller than $K$, a contradiction. Therefore, $t_{x}>s_{y}$. If $s_{x} \geq s_{y}$ then the paths $Q, x-h_{s_{x}}-P_{1}-h_{s_{y}}-y$ and $x-h_{t_{x}}-P_{2}-b_{2}-b_{1}-P_{1}-f_{r+1^{-}} y$ form a theta, $K^{\prime}$. Since $V\left(K^{\prime}\right) \subseteq V(K) \cup\{x, y\} \backslash\left\{a_{1}, a_{2}, a_{3}\right\}, K^{\prime}$ is smaller than $K$, a contradiction. Therefore, $s_{x}<s_{y}$. If $x$ is not adjacent to $h_{s_{y}}$, then the path $h_{s_{y}}-y-f_{r}-x$, a path between $h_{s_{y}}$ and $x$ with interior in $V\left(h_{s_{y}}-P_{2}-h_{s_{x}}\right)$ and a path between $h_{s_{y}}$ and $x$ with interior in $V\left(h_{s_{y}}-P_{2}-h_{t_{x}}\right)$ form a theta smaller than $K$, a contradiction. Thus $x$ is adjacent to $h_{s y}$, and $y$ is a broom, so the theorem holds. This proves (2).

(3) If neither $x$ nor $y$ has a neighbor in $V(A) \cap V\left(P_{1}\right)$, then the theorem holds.

Suppose that neither $x$ nor $y$ has a neighbor in $V(A) \cap V\left(P_{1}\right)$. By 4.2, each of $x$ and $y$ is either complete or anticomplete to $\left\{f_{r-1}, f_{r+1}\right\}$. If both $x$ and $y$ are $\left\{f_{r-1}, f_{r+1}\right\}$-complete, then the paths $x-f_{r-1}-y, x-f_{r+1}-y$ and a path between $x$ and $y$ with interior in $P_{2}^{*}$ form a theta smaller than $K$, a contradiction. This means that at most one of $x$ and $y$ is $\left\{f_{r-1}, f_{r+1}\right\}$ complete. 
Assume that exactly one of $x$ and $y$ is $\left\{f_{r-1}, f_{r+1}\right\}$-complete. By symmetry, we may assume that $x$ is, and that $y$ is anticomplete to $\left\{f_{r-1}, f_{r+1}\right\}$. By 4.1 and $4.2,\left|N(y) \cap V\left(P_{2}\right)\right| \geq 3$, so $s_{y}+1<t_{y}$.

Suppose that $\left|N(x) \cap V\left(P_{2}\right)\right|>1$; then by $4.2,\left|N(x) \cap V\left(P_{2}\right)\right| \geq 3$ so $s_{x}+1<t_{x}$. If there are two paths $S$ and $R$ between $x$ and $y$ whose interiors are anticomplete to each other and contained in $P_{2}^{*}$, then $S, R$ and $x-f_{r}-y$ form a theta smaller than $K$, a contradiction. So from 4.3, we may assume that $t_{x} \leq s_{y}+1$. From this it follows that $s_{x}<s_{y}$. Then the triangles $\left\{f_{r}, f_{r+1}, x\right\}$ and $\left\{b_{1}, b_{2}, b_{3}\right\}$ and the paths $f_{r}-y-h_{t_{y}}-P_{2}-b_{2}, f_{r+1}-P_{1}-b_{1}$ and $x-h_{s_{x}}-P_{2}-a_{2}-a_{3}-P_{3}-b_{3}$ form a prism, $K^{\prime}$. Since $V\left(K^{\prime}\right) \subseteq V(K) \cup\{x, y\} \backslash$ $\left\{a_{1}, h_{s_{y}}, f_{r-1}\right\}, K^{\prime}$ is smaller than $K$, a contradiction. This proves that $\left|N(x) \cap V\left(P_{2}\right)\right|=1$.

If $s_{x}<s_{y}$ then the triangles $\left\{f_{r-1}, f_{r}, x\right\}$ and $\left\{a_{1}, a_{2}, a_{3}\right\}$ and the paths $f_{r-1}-P_{1}-a_{1}, f_{r}-y-h_{t_{y}}-P_{2}-b_{2}-b_{3}-P_{3}-a_{3}$ and $x-h_{s_{x}}-P_{2}-a_{2}$ form a prism, $K^{\prime}$. Since $V\left(K^{\prime}\right) \subseteq V(K) \cup\{x, y\} \backslash\left\{h_{s_{y}}, b_{1}, f_{r+1}\right\}, K^{\prime}$ is smaller than $K$, a contradiction. Therefore $s_{x} \geq s_{y}$, and from the symmetry $s_{x} \leq t_{y}$.

If $y$ is not adjacent to $h_{s_{x}}$, then $s_{y}<s_{x}$ and $t_{y}>s_{x}$. The path $y-f_{r}-x-h_{s_{x}}$ and two paths between $y$ and $h_{s_{x}}$ with interiors in $V\left(h_{s_{y}}-P_{2}-h_{s_{x}}\right)$ and $V\left(h_{t_{y}}-P_{2}-h_{s_{x}}\right)$ form a theta smaller than $K$, a contradiction. So $y$ is adjacent to $h_{s_{x}}$. Since $x$ is a broom, the theorem holds.

Since we have shown that the theorem holds when one of $x$ and $y$ is $\left\{f_{r-1}, f_{r+1}\right\}$-complete, we may now assume that $\{x, y\}$ is anticomplete to $\left\{f_{r-1}, f_{r+1}\right\}$. Now $N(x) \cap V\left(P_{1}\right)=N(y) \cap V\left(P_{1}\right)=\left\{f_{r}\right\}$, so by 4.1 and 4.2 , $x$ and $y$ each have two nonadjacent neighbors in $P_{2}$. If there are two paths $S$ and $R$ between $x$ and $y$ whose interiors are anticomplete to each other and contained in $P_{2}^{*}$, then the paths $S, R$ and $x$ - $f_{r}-y$ form a theta smaller than $K$, a contradiction. Therefore, by 4.3 , we may assume that $t_{x} \leq s_{y}+1$.

If $t_{x} \leq s_{y}$ then the paths $x-f_{r}-y, x-h_{t_{x}}-P_{2}-h_{s_{y}}-y$ and $x-h_{s_{x}}-P_{2}-a_{2}-a_{3}-P_{3}-b_{3}-b_{2}-P_{2}-h_{t_{y}}-y$ form a theta, $K^{\prime}$. Since $V\left(K^{\prime}\right) \subseteq V(K) \cup\{x, y\} \backslash\left\{a_{1}, b_{1}, h_{s_{y}+1}\right\}, K^{\prime}$ is smaller than $K$, a contradiction. Therefore $t_{x}>s_{y}$ so $t_{x}=s_{y}+1$.

If $s_{x}<s_{y}-1$ and $t_{y}>t_{x}+1$ then the paths $x-f_{r}-y, x-h_{s_{x}}-P_{2}-a_{2}-a_{3}-P_{3}-b_{3}-b_{2}-P_{2}-h_{t_{y}}-y$ and a path between $x$ and $y$ with interior in $\left\{h_{s_{y}}, h_{t_{x}}\right\}$ form a theta, $K^{\prime}$. Since $V\left(K^{\prime}\right) \subseteq V(K) \cup\{x, y\} \backslash\left\{a_{1}, b_{1}, h_{s_{y}-1}\right\}, K^{\prime}$ is smaller than $K$, a contradiction. Therefore, either $s_{x} \geq s_{y}-1$ or $t_{y} \leq t_{x}+1$. From the symmetry we may assume that $s_{x} \geq s_{y}-1$. Then since $s_{x}<t_{x}-1$ and $t_{x}=s_{y}+1$, it follows that $s_{x}=s_{y}-1$. If $x$ is not adjacent to $h_{s_{y}}$ then $\left|N(x) \cap V\left(P_{2}\right)\right|=2$, contradicting 4.2. So $x$ is adjacent to $h_{s_{y}}$. Then $x$ is a broom and the theorem holds. This proves (3).

Now 4.7 follows from (1), (2) and (3). 
Let $M$ be the subgraph of $G$ induced on the set of $K$-major vertices. The following algorithm constructs a superset of $V(M)$ that is disjoint from $V(K)$ and is used for cleaning.

4.8. There is an algorithm with the following specifications:

- Input: A graph $G$.

- Output: A sequence of subsets $X_{1}, \ldots, X_{r}$ of $V(G)$, with $r \leq 2|V(G)|^{6}$, such that for every smallest prism $K$ in $G$, if $K$ has a tidy frame then one of $X_{1}, \ldots, X_{r}$ is disjoint from $V(K)$ and contains all $K$-major vertices.

- Running Time: $O\left(|V(G)|^{7}\right)$.

Proof. The algorithm is as follows. For each triple of vertices $(a, b, c)$ compute the set $U$ of vertices complete to $N(a, b) \cup\{c\}$. List all subsets $W \subseteq$ $V(G)$ of at most three vertices. For each pair of $(a, b, c)$ and $W$, compute the subset

$$
N(a, b) \cup\{c\} \cup U \backslash W .
$$

Label the subsets generated $Y_{1}, \ldots, Y_{s}$, and label the list of subsets $N(a, b)$ by $N_{1}, \ldots, N_{p}$. Enumerate all quintuples of vertices $\left(u, w, z_{1}, z_{2}, z_{3}\right)$. For each, compute the subset

$Z\left(u, w, z_{1}, z_{2}, z_{3}\right)=\{u\} \cup\left(N(u) \backslash\left\{w, z_{1}, z_{2}, z_{3}\right\}\right) \cup N\left(w, z_{1}\right) \cup N\left(w, z_{2}\right) \cup N\left(w, z_{3}\right)$.

Label the subsets generated $Z_{1}, \ldots, Z_{t}$. Output the subsets

$$
\emptyset, N_{1}, \ldots, N_{p}, Y_{1}, \ldots, Y_{s}, Z_{1}, \ldots, Z_{t} .
$$

This completes the description of the algorithm.

Since $p \leq|V(G)|^{2}, s \leq|V(G)|^{6}$ and $t \leq|V(G)|^{5}$, the number of subsets in the output sequence is $r \leq 2|V(G)|^{6}$. For each triple $(a, b, c)$, the subset $U$ can be computed in quadratic time and the subsets $W$ can be enumerated in cubic time. Computing $N(a, b) \cup\{c\} \cup U \backslash W$ takes linear time, so the time taken to generate the sequence $N_{1}, \ldots, N_{p}, Y_{1}, \ldots, Y_{s}$ is $O\left(|V(G)|^{7}\right)$. Since each of the $|V(G)|^{5}$ subsets $Z$ can be computed in linear time, their computation does not affect the total running time.

Let $K$ be a smallest prism in $G$, and assume $K$ has a tidy frame. Let $M$ be the subgraph induced by $G$ on the set of $K$-major vertices. Suppose $M$ is the complete graph. We may assume that there exists a set $\{a, b\} \subseteq V(K)$ that is not a subset of the vertex set of a 3 -vertex subpath of $K$, because if no such choice is possible then $V(M)=\emptyset$, which is in the output. We may 
also assume that, subject to these conditions, $\{a, b\}$ is chosen with $|N(a, b)|$ maximum. Then $N(a, b) \subseteq V(M)$. If $N(a, b)=V(M)$ then $V(M)$ is in the output sequence. Otherwise there is a vertex $c \in V(M) \backslash N(a, b)$. Then since $N(a, b) \cup\{c\} \subseteq V(M)$ and $M$ is a complete graph, the set $U$ of vertices complete to $N(a, b) \cup\{c\}$ contains $V(M) \backslash(N(a, b) \cup\{c\})$; so

$$
V(M) \subseteq N(a, b) \cup\{c\} \cup U .
$$

If $U \cap V(K)$ is not a subset of the vertex set of a 3-vertex path of $K$, then there are two vertices $a^{\prime}, b^{\prime} \in U \cap V(K)$ such that $\left\{a^{\prime}, b^{\prime}\right\}$ is not a subset of the vertex set of a 3 -vertex path of $K$. Since $N(a, b) \cup\{c\} \subseteq N\left(a^{\prime}, b^{\prime}\right)$, this contradicts the choice of $a$ and $b$. So $|U \cap V(K)| \leq 3$ and we can choose $W=U \cap V(K)$. Then the subset $N(a, b) \cup\{c\} \cup U \backslash W$ contains $V(M)$ and since $(N(a, b) \cup\{c\}) \cap V(K)=\emptyset, N(a, b) \cup\{c\} \cup U \backslash W$ is disjoint from $V(K)$. The subset in the output corresponding to this choice of $(a, b, c)$ and $W$ contains $V(M)$ and is disjoint from $V(K)$.

Therefore, we may assume that $M$ is not a complete graph. Then there exist two vertices in $V(M)$ that are not adjacent to each other. By 4.7, one of these is a broom. So we may choose $u$ to be a broom such that $z_{1}, z_{2}, z_{3}$ and $w$ are its neighbors in $K$ and $z_{1}-z_{2}-z_{3}$ is a path of $K$. By 4.7, any nonneighbor of $u$ in $V(M)$ is contained in the subset $N\left(w, z_{1}\right) \cup N\left(w, z_{2}\right) \cup N\left(w, z_{3}\right)$. Therefore the subset $Z\left(u, w, z_{1}, z_{2}, z_{3}\right)$ contains $V(M)$. Since $w$ is in the interior of a different path of the prism $K$ from that containing $\left\{z_{1}, z_{2}, z_{3}\right\}$, the subset $N\left(w, z_{1}\right) \cup N\left(w, z_{2}\right) \cup N\left(w, z_{3}\right)$ is disjoint from $V(K)$. Since $N(u) \cap V(K)=\left\{w, z_{1}, z_{2}, z_{3}\right\}, Z\left(u, w, z_{1}, z_{2}, z_{3}\right)$ is disjoint from $V(K)$. This proves 4.8 .

\section{The Complete Algorithm}

5.1. There is an algorithm with the following specifications:

- Input: A graph $G$.

- Output: Either:

- a theta in $G$, or

- a determination that there is no smallest theta in $G$.

- Running Time: $O\left(|V(G)|^{22}\right)$.

Proof. Here is the algorithm. Enumerate all 11-tuples $\left(a, b, s_{1}, s_{2}, s_{3}, m_{1}, m_{2}, m_{3}, t_{1}, t_{2}, t_{3}\right)$ of vertices of $G$ such that

- $a, b, s_{1}, s_{2}$ and $s_{3}$ are all distinct, 
- $a, b, t_{1}, t_{2}$ and $t_{3}$ are all distinct,

- $a$ is not adjacent to $b$, and

- $a$ is complete to $\left\{s_{1}, s_{2}, s_{3}\right\}$ and $b$ is complete to $\left\{t_{1}, t_{2}, t_{3}\right\}$.

We can find all such 11-tuples in time $O\left(|V(G)|^{11}\right)$. For each 11-tuple do the following.

Remove $N(a) \backslash\left\{s_{1}, s_{2}, s_{3}\right\}$ and $N(b) \backslash\left\{t_{1}, t_{2}, t_{3}\right\}$ from $V(G)$, and for $i=1,2,3$, if $m_{i}$ is adjacent to $a$ and $b$ then remove $N\left(m_{i}\right) \backslash\{a, b\}$ from $V(G)$. This can be done in linear time, and after this step every smallest theta in $G$ with the 11-tuple as its frame has a tidy frame. Run the algorithm of 2.2, which takes $O\left(|V(G)|^{4}\right)$ time. Let $X_{1}, \ldots, X_{t}$ be the subsets in the output; $t \leq|V(G)|^{3}$. For every triple of subsets $\left(X_{i_{1}}, X_{i_{2}}, X_{i_{3}}\right)$ from this list, do the following.

Let $G_{1}$ be the graph induced by $G$ on $V(G) \backslash X_{i_{1}}$. Find shortest paths $S_{1}^{\prime}$ between $s_{1}$ and $m_{1}$ and $T_{1}^{\prime}$ between $m_{1}$ and $t_{1}$ in $G_{1}$. Next, let $G_{2}$ be the graph induced by $G_{1}$ on $V\left(G_{1}\right) \backslash X_{i_{2}}$. Find shortest paths $S_{2}^{\prime}$ between $s_{2}$ and $m_{2}$ and $T_{2}^{\prime}$ between $m_{2}$ and $t_{2}$ in $G_{2}$. Finally, let $G_{3}$ be the graph induced by $G_{2}$ on $V\left(G_{2}\right) \backslash X_{i_{3}}$. Find shortest paths $S_{3}^{\prime}$ between $s_{3}$ and $m_{3}$ and $T_{3}^{\prime}$ between $m_{3}$ and $t_{3}$ in $G_{3}$. Finding these paths takes quadratic time. Finally, test whether the following are true in the graph $G$ :

- For $1 \leq i \leq 3, V\left(S_{i}^{\prime}\right) \backslash\left\{m_{i}\right\}$ and $V\left(T_{i}^{\prime}\right) \backslash\left\{m_{i}\right\}$ are disjoint and anticomplete to each other, and

- For $1 \leq i, j \leq 3$ with $i \neq j, V\left(S_{i}^{\prime}\right) \cup V\left(T_{i}^{\prime}\right)$ and $V\left(S_{j}^{\prime}\right) \cup V\left(T_{j}^{\prime}\right)$ are disjoint and anticomplete to each other.

If these conditions are satisfied, output that $\{a, b\}$ and the three paths $a-s_{1}-S_{1}^{\prime}-m_{1}-T_{1}^{\prime}-t_{1}-b, a-s_{2}-S_{2}^{\prime}-m_{2}-T_{2}^{\prime}-t_{2}-b$ and $a-s_{3}-S_{3}^{\prime}-m_{3}-T_{3}^{\prime}-t_{3}-b$ form a theta and stop. Testing these conditions takes quadratic time. So the time it takes to examine one 11-tuple is $O\left(|V(G)|^{11}\right)$.

After examining all 11-tuples, output that $G$ contains no smallest theta. The total running time is $O\left(|V(G)|^{22}\right)$. Now we need to prove that this algorithm is correct. Suppose there is a smallest theta $K$ in $G$; let its halfpaths be $S_{1}, T_{1}, S_{2}, T_{2}, S_{3}$ and $T_{3}$. Some 11-tuple chosen is the frame of $K$, and after the first step of the algorithm, $K$ and every smallest theta in $G$ with the same frame as $K$ have a tidy frame.

By 2.2, we may choose $X_{i_{1}}$ such that the graph $G_{1}$ contains $K$ and there are no bad shortcuts across $S_{1}$ or $T_{1}$ in $G_{1}$. Therefore, the subgraph induced by $G_{1}$ on $V(K) \backslash\left(V\left(S_{1}\right) \cup V\left(T_{1}\right)\right) \cup V\left(S_{1}^{\prime}\right) \cup V\left(T_{1}^{\prime}\right)$ is a theta, $K_{1}$, in $G_{1}$. Since $S_{1}^{\prime}$ and $T_{1}^{\prime}$ are shortest paths, $\left|V\left(K_{1}\right)\right| \leq|V(K)|$, so $K_{1}$ is also a smallest 
theta in $G_{1}$. Since $K_{1}$ is not smaller than $K$ in $G$ it follows that $V\left(S_{1}^{\prime}\right) \backslash\left\{m_{1}\right\}$ and $V\left(T_{1}^{\prime}\right) \backslash\left\{m_{1}\right\}$ are anticomplete to each other. We observe that $K_{1}$ has the same frame as $K$, so it has a tidy frame.

Therefore, by 2.2, for some subset $X_{i_{2}}$ the graph $G_{2}$ contains $K_{1}$ and all shortcuts across $S_{2}$ or $T_{2}$ in $G_{2}$ are good shortcuts across $K_{1}$. Then the subgraph induced by $G_{2}$ on $V\left(K_{1}\right) \backslash\left(V\left(S_{2}\right) \cup V\left(T_{2}\right)\right) \cup V\left(S_{2}^{\prime}\right) \cup V\left(T_{2}^{\prime}\right)$ is a theta, $K_{2}$, in $G_{2}$. Since $S_{2}^{\prime}$ and $T_{2}^{\prime}$ are shortest paths, $\left|V\left(K_{2}\right)\right| \leq\left|V\left(K_{1}\right)\right|$, so $K_{2}$ is also a smallest theta in $G_{2}$. Since $K_{2}$ is not smaller than $K_{1}$ in $G$ it follows that $V\left(S_{2}^{\prime}\right) \backslash\left\{m_{2}\right\}$ and $V\left(T_{2}^{\prime}\right) \backslash\left\{m_{2}\right\}$ are anticomplete to each other. We observe that $K_{2}$ has the same frame as $K$, so it has a tidy frame.

Finally, by 2.2 again, for some subset $X_{i_{3}}$ the graph $G_{3}$ contains $K_{2}$ and all shortcuts across $S_{3}$ or $T_{3}$ in $G_{3}$ are good shortcuts across $K_{2}$. Then the subgraph induced by $G_{3}$ on $V\left(K_{2}\right) \backslash\left(V\left(S_{3}\right) \cup V\left(T_{3}\right)\right) \cup V\left(S_{3}^{\prime}\right) \cup V\left(T_{3}^{\prime}\right)$ is a theta, $K_{3}$, in $G_{3}$. Since $K_{3}$ is not smaller than $K_{2}$ in $G$ it follows that $V\left(S_{3}^{\prime}\right) \backslash\left\{m_{3}\right\}$ and $V\left(T_{3}^{\prime}\right) \backslash\left\{m_{3}\right\}$ are anticomplete to each other. We observe that $K_{3}$ is the theta that is output by the algorithm when it considers $X_{i_{1}}, X_{i_{2}}, X_{i_{3}}$. This proves that the output of the algorithm is a theta.

Conversely, if the algorithm outputs that there is a theta, then the properties of the 11-tuple and the conditions on the paths that the algorithm checks ensure that this output is actually a theta.

5.2. There is an algorithm with the following specifications:

- Input: $A$ graph $G$.

- Output: Either:

- a prism in $G$, or

- a determination that there is no smallest prism in $G$.

- Running Time: $O\left(|V(G)|^{35}\right)$.

Proof. Here is the algorithm. Enumerate all 15-tuples of vertices of $G$

$$
\left(a_{1}, a_{2}, a_{3}, m_{1}, m_{2}, m_{3}, b_{1}, b_{2}, b_{3}, a_{1}^{\prime}, a_{2}^{\prime}, a_{3}^{\prime}, b_{1}^{\prime}, b_{2}^{\prime}, b_{3}^{\prime}\right)
$$

such that

- $a_{1}, a_{2}, a_{3}, b_{1}, b_{2}, b_{3}$ are distinct,

- $a_{1}, a_{2}, a_{3}, a_{1}^{\prime}, a_{2}^{\prime}, a_{3}^{\prime}$ are distinct,

- $b_{1}, b_{2}, b_{3}, b_{1}^{\prime}, b_{2}^{\prime}, b_{3}^{\prime}$ are distinct,

- $G$ induces a triangle on each of $\left\{a_{1}, a_{2}, a_{3}\right\}$ and $\left\{b_{1}, b_{2}, b_{3}\right\}$, and

- for $i=1,2,3, a_{i}^{\prime}$ is adjacent to $a_{i}$ and $b_{i}^{\prime}$ is adjacent to $b_{i}$. 
For each 15-tuple, do the following.

For $i=1,2,3$ remove the subsets $N\left(a_{i}\right) \backslash\left\{a_{i}^{\prime}, a_{1}, a_{2}, a_{3}\right\}$ and $N\left(b_{i}\right) \backslash$ $\left\{b_{i}^{\prime}, b_{1}, b_{2}, b_{3}\right\}$ from $V(G)$, and if $m_{i}=a_{i}^{\prime}=b_{i}^{\prime}$ then remove $N\left(m_{i}\right) \backslash\left\{a_{i}, b_{i}\right\}$ from $V(G)$. Now any smallest prism $K$ that has $\left\{a_{1}, a_{2}, a_{3}, m_{1}, m_{2}, m_{3}, b_{1}, b_{2}, b_{3}\right\}$ as its frame and contains $\left\{a_{1}^{\prime}, a_{2}^{\prime}, a_{3}^{\prime}, b_{1}^{\prime}, b_{2}^{\prime}, b_{3}^{\prime}\right\}$ has a tidy frame.

Run the algorithm of 4.8 on $G$. This outputs $O\left(|V(G)|^{6}\right)$ subsets. For each subset $Y$ output, do the following. Let $G^{\prime}$ be the graph induced by $G$ on $V(G) \backslash Y$. Run the algorithm of 3.5, which takes $O\left(|V(G)|^{5}\right)$ time and outputs $O\left(|V(G)|^{4}\right)$ subsets. Let $X_{1}, \ldots, X_{t}$ be the subsets in the output. For every triple of subsets $\left(X_{i_{1}}, X_{i_{2}}, X_{i_{3}}\right)$ from this list, do the following.

Let $G_{1}$ be the graph induced by $G^{\prime}$ on $V\left(G^{\prime}\right) \backslash X_{i_{1}}$. Find shortest paths $S_{1}^{\prime}$ between $a_{1}$ and $m_{1}$ and $T_{1}^{\prime}$ between $m_{1}$ and $b_{1}$ in $G_{1}$. Remove from $V\left(G_{1}\right)$ the set $U_{1}$ consisting of all vertices in $V\left(G_{1}\right) \backslash\left(S_{1}^{\prime *} \cup T_{1}^{* *} \cup\left\{a_{1}, b_{1}, m_{1}\right\}\right)$ that have a neighbor in $S_{1}^{\prime *} \cup T_{1}^{* *}$. Next, let $G_{2}$ be the graph induced by $G_{1}$ on $V\left(G_{1}\right) \backslash X_{i_{2}}$. Find shortest paths $S_{2}^{\prime}$ between $a_{2}$ and $m_{2}$ and $T_{2}^{\prime}$ between $m_{2}$ and $b_{2}$ in $G_{2}$. Remove from $G_{2}$ the set $U_{2}$ consisting of all vertices in $V\left(G_{2}\right) \backslash\left(S_{2}^{\prime *} \cup T_{2}^{\prime *} \cup\left\{a_{2}, b_{2}, m_{2}\right\}\right)$ that have a neighbor in $S_{2}^{* *} \cup T_{2}^{\prime *}$. Finally, let $G_{3}$ be the graph induced by $G_{2}$ on $V\left(G_{2}\right) \backslash X_{i_{3}}$. Find shortest paths $S_{3}^{\prime}$ between $a_{3}$ and $m_{3}$ and between $m_{3}$ and $b_{3}$ in $G_{3}$. Finding these paths and removing these subsets takes quadratic time.

Finally, for $1 \leq i<j \leq 3$ test whether $V\left(S_{i}^{\prime}\right) \cup V\left(T_{i}^{\prime}\right) \cup V\left(S_{j}^{\prime}\right) \cup V\left(T_{j}^{\prime}\right)$ is the vertex set of an induced cycle in the graph $G$. If so, then output that the triangles $\left\{a_{1}, a_{2}, a_{3}\right\}$ and $\left\{b_{1}, b_{2}, b_{3}\right\}$ and the paths $a_{1}-S_{1}^{\prime}-m_{1}-T_{1}^{\prime}-b_{1}$, $a_{2}-S_{2}^{\prime}-m_{2}-T_{2}^{\prime}-b_{2}$ and $a_{3}-S_{3}^{\prime}-m_{3}-T_{3}^{\prime}-b_{3}$ form a prism and stop. Testing this takes quadratic time. So the time it takes to examine one 15-tuple is $O\left(|V(G)|^{20}\right)$.

After examining all 15-tuples, output that $G$ contains no smallest prism. The total running time is $O\left(|V(G)|^{35}\right)$.

Now we need to prove that this algorithm is correct. Suppose there is a smallest prism $K$ in $G$; let its half-paths be $S_{1}, T_{1}, S_{2}, T_{2}, S_{3}$ and $T_{3}$. For some 15-tuple chosen, $\left\{a_{1}, a_{2}, a_{3}, m_{1}, m_{2}, m_{3}, b_{1}, b_{2}, b_{3}\right\}$ is the frame of $K$, and $\left\{a_{1}^{\prime}, a_{2}^{\prime}, a_{3}^{\prime}, b_{1}^{\prime}, b_{2}^{\prime}, b_{3}^{\prime}\right\} \subseteq V(K)$. Therefore, after the first step of the algorithm runs for this 15 -tuple, $K$ has a tidy frame.

(1) Let $J$ be an almost clean smallest prism in a graph $H$ and let $S^{\prime}$ be a good shortcut across a half-path $S$ of $J$. Let $U \subset V(H)$ be the set of vertices not in $V\left(S^{\prime}\right)$ that have a neighbor in $S^{\prime *}$. Then the subgraph $J^{\prime}$ induced by $H$ on $(V(J) \backslash V(S)) \cup V\left(S^{\prime}\right)$ is an almost clean smallest prism in the graph induced by $H$ on $V(H) \backslash U$.

Since $S^{\prime}$ is a good shortcut, the subgraph $J^{\prime}$ induced by $H$ on $(V(J) \backslash$ $V(S)) \cup V\left(S^{\prime}\right)$ is a prism. Since a good shortcut is a shortest path, $\left|V\left(J^{\prime}\right)\right| \leq$ 
$|V(J)|$, so $J^{\prime}$ is a smallest prism in $H$. We observe that since $J^{\prime}$ has the same frame as $J$, it has a tidy frame. Assume that there is a $J^{\prime}$-major vertex $v \in V(H) \backslash U$. Then since $v \notin U, N(v) \cap S^{* *}=\emptyset$. Since $v$ is $J^{\prime}$-major, this means that $N(v) \cap\left(V\left(J^{\prime}\right) \backslash S^{*}\right)$ is not a subset of the vertex set of a 3-vertex path in $J^{\prime}$. Since $N(v) \cap\left(V\left(J^{\prime}\right) \backslash S^{*}\right)=N(v) \cap\left(V(J) \backslash S^{*}\right), v$ is $J$-major, contradicting the fact that $J$ is almost clean in $H$. Therefore, all $J^{\prime}$-major vertices are contained in $U$. We observe also that $U$ is disjoint from $V\left(J^{\prime}\right)$ because $J^{\prime}$ is a prism, so $J^{\prime}$ is an almost clean prism in the the graph induced by $H$ on $V(H) \backslash U$. This proves (1).

By 4.8, we can choose $Y$ such that $G^{\prime}$ contains no $K$-major vertices. Then $K$ is almost clean in $G^{\prime}$, so by 3.5 , we can choose $X_{i_{1}}$ such that the graph $G_{1}$ contains $K$ and there are no bad shortcuts across $S_{1}$ or $T_{1}$ in $G_{1}$. Let $K_{1}$ be the subgraph induced by $G_{1}$ on $V(K) \backslash\left(V\left(S_{1}\right) \cup V\left(T_{1}\right)\right) \cup V\left(S_{1}^{\prime}\right) \cup V\left(T_{1}^{\prime}\right)$. Since $K_{1}$ is not smaller than $K$ in $G, S_{1}^{*}$ is anticomplete to $T_{1}^{* *}$. It now follows from (1) applied twice (once for each half-path $S_{1}$ and $T_{1}$ ) that after removing the set $U_{1}$ from $V\left(G_{1}\right), K_{1}$ is an almost clean smallest prism in $G_{1}$.

Therefore, by 3.5 , we can choose $X_{i_{2}}$ such that the graph $G_{2}$ contains $K_{1}$ and there are no bad shortcuts across $S_{2}$ or $T_{2}$ in $G_{2}$. Let $K_{2}$ be the subgraph induced by $G_{2}$ on $V\left(K_{1}\right) \backslash\left(V\left(S_{2}\right) \cup V\left(T_{2}\right)\right) \cup V\left(S_{2}^{\prime}\right) \cup V\left(T_{2}^{\prime}\right)$. Since $K_{2}$ is not smaller than $K_{1}$ in $G, S_{2}^{* *}$ is anticomplete to $T_{2}^{\prime *}$. Again it follows from (1) applied twice (once for each half-path $S_{2}$ and $T_{2}$ ) that after removing the set $U_{2}$ from $V\left(G_{2}\right), K_{2}$ is an almost clean smallest prism in $G_{2}$.

Finally, by 3.5 , we can choose $X_{i_{3}}$ such that the graph $G_{3}$ contains $K_{2}$ and there are no bad shortcuts across $S_{3}$ or $T_{3}$ in $G_{3}$. Then it follows that the subgraph $K_{3}$ induced by $G_{3}$ on $V\left(K_{2}\right) \backslash\left(V\left(S_{3}\right) \cup V\left(T_{3}\right)\right) \cup V\left(S_{3}^{\prime}\right) \cup V\left(T_{3}^{\prime}\right)$ is a prism. Since $K_{3}$ is not smaller than $K_{2}$ in $G, S_{3}^{\prime *}$ is anticomplete to $T_{3}^{\prime *}$. We observe that since $G_{3}$ is an induced subgraph of $G$, a prism in $G_{3}$ is a prism in $G$, and that $K_{3}$ is the prism output by the algorithm when it considers $X_{i_{1}}, X_{i_{2}}, X_{i_{3}}$. This proves that the algorithm outputs a prism.

Conversely, if the algorithm outputs that there is a prism, then the properties of the 15-tuple and the conditions on the paths that the algorithm checks ensure that this output is actually a prism.

Finally, we can put together the algorithms of 5.1 and 5.2 to construct the complete algorithm.

5.3. There is an algorithm with the following specifications:

- Input: A graph $G$. 
- Output: Either:

- a theta or a prism in $G$, or

- a determination that there is no theta or prism in $G$.

- Running Time: $O\left(|V(G)|^{35}\right)$.

Proof. First run the algorithm of 5.1. If it outputs a theta then output this theta and stop. Otherwise run the algorithm of 5.2. If it outputs a prism then output this prism and stop. Otherwise, output that there is no theta or prism in $G$.

The running time of this algorithm is the maximum of those of 5.1 and 5.2 , which is $O\left(|V(G)|^{35}\right)$. If this algorithm outputs either a theta or a prism, then it follows from 5.1 and 5.2 that it is correct. Conversely, if $G$ contains a theta or a prism, then it contains a smallest theta or a smallest prism. Therefore, one of the algorithms of 5.1 or of 5.2 will output a theta or a prism, which will then be output by this algorithm. This proves 5.3.

\section{References}

[1] M. Chudnovsky, G. Cornuéjols, X. Liu, P. Seymour, and K. Vušković, "Recognizing Berge graphs", Combinatorica, 25 (2005), no. 2, 143-186.

[2] M. Chudnovsky and P. Seymour, "The three-in-a-tree problem", submitted for publication.

[3] M. Conforti and M. R. Rao, "Testing balancedness and perfection of linear matrices", Mathematical Programming 61 (1993), 1-18.

[4] F. Maffray and N. Trotignon, "Algorithms for perfectly contractile graphs", SIAM J. Discrete Math. 19 (2005), no. 3, 553-574. 